JOURNAL OF THE AMERICAN MATHEMATICAL SOCIETY

Volume 17, Number 1, Pages 197-227

S 0894-0347(03)00440-5

Article electronically published on October 3, 2003

\title{
THE TAME AND THE WILD AUTOMORPHISMS OF POLYNOMIAL RINGS IN THREE VARIABLES
}

IVAN P. SHESTAKOV AND UALBAI U. UMIRBAEV

\section{INTRODUCTION}

Let $C=F\left[x_{1}, x_{2}, \ldots, x_{n}\right]$ be the polynomial ring in the variables $x_{1}, x_{2}, \ldots, x_{n}$ over a field $F$, and let $A u t C$ be the group of automorphisms of $C$ as an algebra over $F$. An automorphism $\tau \in A u t C$ is called elementary if it has a form

$$
\tau:\left(x_{1}, \ldots, x_{i-1}, x_{i}, x_{i+1}, \ldots, x_{n}\right) \mapsto\left(x_{1}, \ldots, x_{i-1}, \alpha x_{i}+f, x_{i+1}, \ldots, x_{n}\right),
$$

where $0 \neq \alpha \in F, f \in F\left[x_{1}, \ldots, x_{i-1}, x_{i+1}, \ldots, x_{n}\right]$. The subgroup of AutC generated by all the elementary automorphisms is called the tame subgroup, and the elements from this subgroup are called tame automorphisms of $C$. Non-tame automorphisms of the algebra $C$ are called wild.

It is well known [6], [9], 10, 11] that the automorphisms of polynomial rings and free associative algebras in two variables are tame. At present, a few new proofs of these results have been found (see [5], [8]). However, in the case of three or more variables the similar question was open and known as "The generation gap problem" 2], 3] or "Tame generators problem" 8]. The general belief was that the answer is negative, and there were several candidate counterexamples (see [5], 8], [12, [7], [19]). The best known of them is the following automorphism $\sigma \in \operatorname{Aut}(F[x, y, z])$, constructed by Nagata in 1972 (see [12]):

$$
\begin{aligned}
& \sigma(x)=x+\left(x^{2}-y z\right) z, \\
& \sigma(y)=y+2\left(x^{2}-y z\right) x+\left(x^{2}-y z\right)^{2} z, \\
& \sigma(z)=z .
\end{aligned}
$$

Observe that the Nagata automorphism is stably tame [17]; that is, it becomes tame after adding new variables.

The purpose of the present work is to give a negative answer to the above question. Our main result states that the tame automorphisms of the polynomial ring $A=F[x, y, z]$ over a field $F$ of characteristic 0 are algorithmically recognizable. In particular, the Nagata automorphism $\sigma$ is wild.

The approach we use is different from the traditional ones. The novelty consists of the imbedding of the polynomial ring $A$ into the free Poisson algebra (or the

Received by the editors January 8, 2003

2000 Mathematics Subject Classification. Primary 13F20, 13P10, 14H37; Secondary 14R10, $14 \mathrm{R} 15$.

Key words and phrases. Rings of polynomials, automorphisms, subalgebras.

The first author was supported by CNPq.

The second author was supported by the FAPESP Proc.00/06832-8. 
algebra of universal Poisson brackets) on the same set of generators and of the systematical use of brackets as an additional tool.

The crucial role in the proof is played by the description of the structure of subalgebras generated by so-called $*$-reduced pairs of polynomials, given in [16]. More precisely, a lower estimate for degrees of elements of these subalgebras is essentially used in most of the proofs.

We follow the so-called "method of simple automorphisms", which was first developed in [1 for a characterization of tame automorphisms of two-generated free Leibniz algebras. Note that this method permits us also to establish directly the result of [4] concerning wild automorphisms of two-generated free matrix algebras, without using the results of [6], 9], [10], [11. In fact, the first attempt to apply this method for a characterization of tame automorphisms of polynomial rings and free associative algebras in three variables was done by C. K. Gupta and U. U. Umirbaev in 1999. At that time, some results were obtained modulo a certain conjecture, which eventually proved not to be true for polynomial rings (see Example 1 , Section 3). Really, the structure of tame automorphisms turns out to be much more complicated.

Observe that no analogues of the results of [16] are known for free associative algebras and for polynomial rings of positive characteristic, and the question on the existence of wild automorphisms is still open for these algebras.

The paper is organized as follows. In Section 2, some results are given, mainly from [16, which are necessary in the sequel. Some instruments for further calculations are also created here. In Section 3, elementary reductions and reductions of types I-IV are defined and characterized for automorphisms of the algebra $A$, and simple automorphisms of $A$ are defined. The main part of the work, Section 4, is devoted to the proof of Theorem 1 which states that every tame automorphism of the algebra $A$ is simple. The main results are formulated and proved in Section 5 as corollaries of Theorem [1.

\section{Structure of two-generated SUbalgebras}

Let $F$ be an arbitrary field of characteristic 0 , and let $A=F\left[x_{1}, x_{2}, x_{3}\right]$ be the ring of polynomials in the variables $x_{1}, x_{2}, x_{3}$ over $F$. Following [16], we will identify $A$ with a certain subspace of the free Poisson algebra $P=P L\left\langle x_{1}, x_{2}, x_{3}\right\rangle$.

Recall that a vector space $B$ over a field $F$, endowed with two bilinear operations $x \cdot y$ (a multiplication) and $[x, y]$ (a Poisson bracket), is called a Poisson algebra if $B$ is a commutative associative algebra under $x \cdot y, B$ is a Lie algebra under $[x, y]$, and $B$ satisfies the Leibniz identity

$$
[x \cdot y, z]=[x, z] \cdot y+x \cdot[y, z] .
$$

An important class of Poisson algebras is given by the following construction. Let $L$ be a Lie algebra with a linear basis $l_{1}, l_{2}, \ldots, l_{k}, \ldots$. Denote by $P(L)$ the ring of polynomials on the variables $l_{1}, l_{2}, \ldots, l_{k}, \ldots$ The operation $[x, y]$ of the algebra $L$ can be uniquely extended to a Poisson bracket $[x, y]$ on the algebra $P(L)$ by means of formula (11), and $P(L)$ becomes a Poisson algebra [15].

Now let $L$ be a free Lie algebra with free generators $x_{1}, x_{2}, \ldots, x_{n}$. Then $P(L)$ is a free Poisson algebra [15] with the free generators $x_{1}, x_{2}, \ldots, x_{n}$. We will denote this algebra by $P L\left\langle x_{1}, x_{2}, \ldots, x_{n}\right\rangle$. If we choose a homogeneous basis

$$
x_{1}, x_{2}, \ldots, x_{n},\left[x_{1}, x_{2}\right], \ldots,\left[x_{1}, x_{n}\right], \ldots,\left[x_{n-1}, x_{n}\right],\left[\left[x_{1}, x_{2}\right], x_{3}\right] \ldots
$$


of the algebra $L$ with nondecreasing degrees, then $P L\left\langle x_{1}, x_{2}, \ldots, x_{n}\right\rangle$, as a vector space, coincides with the ring of polynomials on these elements. The space $P L\left\langle x_{1}, x_{2}, \ldots, x_{n}\right\rangle$ is graded by degrees on $x_{i}$, and for every element $f \in$ $P L\left\langle x_{1}, x_{2}, \ldots, x_{n}\right\rangle$, the highest homogeneous part $\bar{f}$ and the degree $\operatorname{deg} f$ can be defined in an ordinary way. Note that

$$
\overline{f g}=\bar{f} \bar{g}, \quad \operatorname{deg}(f g)=\operatorname{deg} f+\operatorname{deg} g, \quad \operatorname{deg}[f, g] \leq \operatorname{deg} f+\operatorname{deg} g .
$$

In the sequel, we will identify the ring of polynomials $A=F\left[x_{1}, x_{2}, x_{3}\right]$ with the subspace of the algebra $P L\left\langle x_{1}, x_{2}, x_{3}\right\rangle$ generated by elements

$$
x_{1}^{r_{1}} x_{2}^{r_{2}} x_{3}^{r_{3}}, \quad r_{i} \geq 0,1 \leq i \leq 3 .
$$

Note that if $f, g \in A$, then

$$
\begin{aligned}
{[f, g] } & =\gamma_{12}\left[x_{1}, x_{2}\right]+\gamma_{23}\left[x_{2}, x_{3}\right]+\gamma_{13}\left[x_{1}, x_{3}\right], \\
\gamma_{i j} & =\frac{\partial f}{\partial x_{i}} \frac{\partial g}{\partial x_{j}}-\frac{\partial g}{\partial x_{i}} \frac{\partial f}{\partial x_{j}}, \quad 1 \leq i<j \leq 3 .
\end{aligned}
$$

If $f_{1}, f_{2}, \ldots, f_{k} \in A$, then by $\left\langle f_{1}, f_{2}, \ldots, f_{k}\right\rangle$ we denote the subalgebra of the algebra $A$ generated by these elements.

The following lemma is proved in [16].

Lemma 1. Let $f, g, h \in A$. Then the following statements are true:

1) $[f, g]=0$ iff $f, g$ are algebraically dependent.

2) Suppose that $f, g, h \notin F$ and $m=\operatorname{deg}[f, g]+\operatorname{deg} h, n=\operatorname{deg}[g, h]+\operatorname{deg} f$, $k=\operatorname{deg}[h, f]+\operatorname{deg} g$. Then $m \leq \max (n, k)$. If $n \neq k$, then $m=\max (n, k)$.

The next two simple statements are well known (see [5]):

F1) If $a, b$ are nonzero homogeneous algebraically dependent elements of $A$, then there exists an element $z \in A$ such that $a=\alpha z^{n}, b=\beta z^{m}, \alpha, \beta \in F$. In addition, the subalgebra $\langle a, b\rangle$ is one-generated iff $m \mid n$ or $n \mid m$.

F2) Let $f, g \in A$ and $\bar{f}, \bar{g}$ are algebraically independent. If $h \in\langle f, g\rangle$, then $\bar{h} \in\langle\bar{f}, \bar{g}\rangle$.

Recall that a pair of elements $f, g$ of the algebra $A$ is called reduced (see [18]), if $\bar{f} \notin\langle\bar{g}\rangle, \bar{g} \notin\langle\bar{f}\rangle$. A reduced pair of algebraically independent elements $f, g \in A$ is called $*$-reduced (see [16]), if $\bar{f}, \bar{g}$ are algebraically dependent.

Let $f, g$ be a $*$-reduced pair of elements of $A$ and $n=\operatorname{deg} f<m=\operatorname{deg} g$. Put $p=\frac{n}{(n, m)}, s=\frac{m}{(n, m)}$,

$$
N=N(f, g)=\frac{m n}{(m, n)}-m-n+\operatorname{deg}[f, g]=m p-m-n+\operatorname{deg}[f, g],
$$

where $(n, m)$ is the greatest common divisor of $n, m$. Note that $(p, s)=1$, and by F1) there exists an element $a \in A$ such that $\bar{f}=\beta a^{p}, \bar{g}=\gamma a^{s}$. Sometimes, we will call a $*$-reduced pair of elements $f, g$ also a $p$-reduced pair. Let $G(x, y) \in F[x, y]$. It was proved in [16] that if $\operatorname{deg}_{y}(G(x, y))=p q+r, 0 \leq r<p$, then

$$
\operatorname{deg}(G(f, g)) \geq q N+m r,
$$

and if $\operatorname{deg}_{x}(G(x, y))=s q_{1}+r_{1}, 0 \leq r_{1}<s$, then

$$
\operatorname{deg}(G(f, g)) \geq q_{1} N+n r_{1} .
$$

It will be convenient for us to collect several evident properties of the $*$-reduced pair $f, g$ in the following lemma. 
Lemma 2. Under the above notation,

i) $p \geq 2$;

ii) $N=N(f, g)>\operatorname{deg}[f, g]$;

iii) if $p>2$, then $N>m$;

iv) if $p=2$, then $N>\frac{n}{2}$.

The properties $i)-i i i)$ are evident. As for $i v)$, let $d=(n, m)$; then $n=2 d, m=$ $s d$, and $N=s d-2 d+\operatorname{deg}[f, g]>(s-2) d \geq d=\frac{n}{2}$.

The statement of the following lemma is easily proved.

Lemma 3. The elements of type $f^{i} g^{j}$, where $j<p$, have different degrees for different values of $i, j$.

Inequality (2) and Lemma 3 imply

Corollary 1. Let $G(x, y) \in F[x, y], h=G(f, g)$. Consider the following conditions:

(i) $\operatorname{deg} h<N(f, g)$;

(ii) $\operatorname{deg}_{y}(G(x, y))<p$;

(iii) $h=\sum_{i, j} \alpha_{i j} f^{i} g^{j}$, where $\alpha_{i j} \in F$ and $i n+j m \leq \operatorname{deg} h$ for all $i, j$;

(iv) $\bar{h} \in\langle\bar{f}, \bar{g}\rangle$.

Then $(i) \Rightarrow(i i) \Rightarrow(i i i) \Rightarrow(i v)$.

Suppose that $p \geq 3$ or $\operatorname{deg}[f, g]>n$. Then obviously $N(f, g)>m$, and in the conditions of Corollary 1 we have $\bar{h} \in\langle\bar{f}, \bar{g}\rangle$ or $\operatorname{deg} h>m=\max (\operatorname{deg} f, \operatorname{deg} g)$. Note that the most complicated case in the investigation of tame automorphisms of $A$ is represented by $*$-reduced pairs $f, g$ with the condition $N(f, g) \leq m$, that is, by 2-reduced pairs $f, g$ for which $\operatorname{deg}[f, g] \leq n$.

Lemma 4. There exists a polynomial $w(x, y) \in F[x, y]$ of the type

$$
w(x, y)=y^{p}-\alpha x^{s}-\sum \alpha_{i j} x^{i} y^{j}, n i+m j<m p,
$$

which satisfies the following conditions:

1) $\operatorname{deg} w(f, g)<p m$;

2) $\overline{w(f, g)} \notin\langle\bar{f}, \bar{g}\rangle$.

Proof. By F1), there exists a homogeneous element $a \in A$ such that $\bar{f}=\beta a^{p}$, $\bar{g}=\gamma a^{s}$. Then there exists $\alpha \in F$ such that $\bar{g}^{p}=\alpha \bar{f}^{s}$, and the elements of the type $\bar{f}^{i} \bar{g}^{j}, j<p$, form a basis of the subalgebra $\langle\bar{f}, \bar{g}\rangle$. Putting $h=g^{p}-\alpha f^{s}$, we have $\operatorname{deg} h<m p$. If $\bar{h} \in\langle\bar{f}, \bar{g}\rangle$, then $\bar{h}=\alpha_{i j} \bar{f}^{i} \bar{g}^{j}$, where $n i+m j<m p$. Change the element $h$ to $h-\alpha_{i j} f^{i} g^{j}$. Then $\operatorname{deg}\left(h-\alpha_{i j} f^{i} g^{j}\right)<\operatorname{deg} h$. After several reductions of this type, we get an element

$$
h=g^{p}-\alpha f^{s}-\sum \alpha_{i j} f^{i} g^{j}, n i+m j<m p,
$$

for which $\bar{h} \notin\langle\bar{f}, \bar{g}\rangle$. Since $f, g$ are algebraically independent, the equality $h=$ $w(f, g)$ defines uniquely a polynomial $w(x, y)$ that satisfies the conditions of the lemma.

A polynomial $w(x, y)$ satisfying the conditions of Lemma 4 we will call a derivative polynomial of the $*$-reduced pair $f, g$. Note that a derivative polynomial $w(x, y)$ is not uniquely defined in the general case. But the coefficient $\alpha$ in the conditions of Lemma 3 is uniquely defined by the equality $\bar{g}^{p}=\alpha \bar{f}^{s}$. 
Lemma 5. Let $w(x, y)$ be a derivative polynomial of a *-reduced pair $f, g$. Then the following statements are true:

1) $\operatorname{deg} w(f, g)$ is uniquely defined;

2) if $\bar{f}, \overline{w(f, g)}$ are algebraically dependent, then $\overline{w(f, g)}$ is uniquely defined;

3) $\operatorname{deg}(w(f, g)) \geq N(f, g)$;

4) if $\operatorname{deg}(w(f, g))<\operatorname{deg} g=m$, then $w(x, y)$ is defined uniquely up to a summand $q(x)$, where $n \cdot \operatorname{deg}(q(x)) \leq \operatorname{deg}(w(f, g))$;

5) if $\operatorname{deg}(w(f, g))<\operatorname{deg} f$, then $w(x, y)$ is defined uniquely up to a scalar summand from $F$.

Proof. Let $w_{1}(x, y)$ be another derivative polynomial of the pair $f, g$. Since the coefficient $\alpha$ is uniquely defined in the conditions of Lemma 4, we have

$$
h(x, y)=w(x, y)-w_{1}(x, y)=\sum \gamma_{i j} x^{i} y^{j},
$$

where $n i+m j<m p$. Now, if $\operatorname{deg}(w(f, g))>\operatorname{deg}\left(w_{1}(f, g)\right)$, then by Lemma 3 we get

$$
\overline{h(f, g)}=\overline{w(f, g)} \in\langle\bar{f}, \bar{g}\rangle,
$$

which contradicts the definition of $w(x, y)$.

Suppose that $\overline{w(f, g)} \neq \overline{w_{1}(f, g)}$. Then $\overline{h(f, g)}=\overline{w(f, g)}-\overline{w_{1}(f, g)}$. Since $\overline{h(f, g)} \in\langle\bar{f}, \bar{g}\rangle$, the elements $\bar{f}, \overline{h(f, g)}$ are algebraically dependent. Now, if $\bar{f}$, $\overline{w(f, g)}$ are algebraically dependent, then $\overline{w(f, g)}, \overline{h(f, g)}$ are algebraically dependent too. Furthermore, since $\operatorname{deg}(w(f, g))=\operatorname{deg}(h(f, g))$, the elements $\overline{w(f, g)}, \overline{h(f, g)}$ are linearly dependent, and thus $\overline{w(f, g)} \in\langle\bar{f}, \bar{g}\rangle$. This again contradicts the definition of $w(x, y)$.

Note that $\operatorname{deg}(h(f, g)) \leq \operatorname{deg}(w(f, g))$. Since $\overline{w(f, g)} \notin\langle\bar{f}, \bar{g}\rangle$, Corollary \yields 3). If $\operatorname{deg}(w(f, g))<\operatorname{deg} g$, then by Lemma 3 we get $h(x, y)=q(x), \operatorname{deg}(q(f))=$ $\operatorname{deg}(h(f, g)) \leq \operatorname{deg}(w(f, g))$. This proves statements 4$), 5)$ of the lemma.

Observe that in view of 3) and Lemma 2,iii), conditions 4), 5) of Lemma 5 may take place only for 2-reduced pairs.

Lemma 6. Let $w(x, y)$ be a derivative polynomial of the pair $f, g$ and $u=w(f, g)$. Then the highest homogeneous parts of the elements of the type

$$
f^{i} g^{j} u^{t}, \quad j<p, \quad 0 \leq t \leq 1,
$$

are linearly independent.

Proof. Assuming the contrary, we get by Lemma 3 the equality of the form

$$
\bar{f}^{i} \bar{g}^{j} \bar{u}=\beta \bar{f}^{i_{1}} \bar{g}^{j_{1}}, \quad j, j_{1}<p .
$$

If $i \leq i_{1}, j \leq j_{1}$, then this equality implies $\bar{u} \in\langle\bar{f}, \bar{g}\rangle$, which is impossible by the definition of $w(x, y)$. Assume that $j \leq j_{1}, i>i_{1}$. Then

$$
\bar{f}^{i-i_{1}} \bar{u}=\beta \bar{g}^{j_{1}-j} .
$$

Since $\bar{u} \notin\langle\bar{f}, \bar{g}\rangle$, by Corollary 1 we have

$$
\operatorname{deg} u \geq N(f, g)=p m-m-n+\operatorname{deg}[f, g] .
$$

Therefore,

$$
\begin{aligned}
\operatorname{deg}\left(\bar{g}^{j_{1}-j}\right)=m\left(j_{1}-j\right) & \geq n\left(i-i_{1}\right)+p m-m-n+\operatorname{deg}[f, g] \\
& \geq(p-1) m+\operatorname{deg}[f, g]>(p-1) m .
\end{aligned}
$$


This contradicts the inequality $j_{1}-j \leq p-1$. If $i \leq i_{1}, j>j_{1}$, then

$$
\bar{g}^{j-j_{1}} \bar{u}=\beta \bar{f}^{i_{1}-i} .
$$

Since $j-j_{1} \leq p-1$ and $\bar{g}^{p}=\alpha \bar{f}^{s}$, we may assume that $i_{1}-i<s$; otherwise $\bar{u} \in\langle\bar{f}, \bar{g}\rangle$. Thus,

$$
\begin{aligned}
\operatorname{deg}\left(f^{i_{1}-i}\right) & =n\left(i_{1}-i\right) \geq m\left(j-j_{1}\right)+p m-m-n+\operatorname{deg}[f, g] \\
& \geq p m-n+\operatorname{deg}[f, g]>p m-n=n s-n=n(s-1),
\end{aligned}
$$

which is impossible.

Lemma 7. Let $w(x, y)$ be a derivative polynomial of the pair $f, g$, and $T(x, y) \in$ $F[x, y], \operatorname{deg}_{y}(T(x, y))<2 p$. Then, the following statements are true:

1) $T(x, y)$ can be uniquely presented in the form

$$
T(x, y)=w(x, y) q(x, y)+s(x, y),
$$

where $\operatorname{deg}_{y}(q(x, y)), \operatorname{deg}_{y}(s(x, y))<p$;

2) if $\operatorname{deg}(T(f, g)) \leq t$, then

$$
\operatorname{deg}(w(f, g))+\operatorname{deg}(q(f, g)) \leq t, \operatorname{deg}(s(f, g)) \leq t ;
$$

3) if $\overline{T(f, g)} \notin\langle\bar{f}, \bar{g}\rangle$, then $q(x, y) \neq 0$ and

$$
\begin{aligned}
& \operatorname{deg}\left(\frac{\partial T}{\partial x}(f, g)\right)=\operatorname{deg}(q(f, g))+n(s-1), \\
& \operatorname{deg}\left(\frac{\partial T}{\partial y}(f, g)\right)=\operatorname{deg}(q(f, g))+m(p-1) .
\end{aligned}
$$

4) if $\overline{T(f, g)} \notin\langle\bar{f}, \bar{g}\rangle$ and $\operatorname{deg}(T(f, g))<\min \{n+N, m p\}$, then $T(x, y)=$ $\lambda w_{1}(f, g), 0 \neq \lambda \in F$, where $w_{1}(x, y)$ is also a derivative polynomial of the pair $f, g$.

Proof. The first statement of the lemma follows from the division algorithm in the ring $(F[x])[y]$; one may divide $T(x, y)$ by $w(x, y)$ since the last polynomial is monic. Furthermore, the right part of the equality $T(f, g)=w(f, g) q(f, g)+s(f, g)$ is a linear combination of elements indicated in Lemma 6 therefore, by this lemma, only the elements of degree less than or equal to $\operatorname{deg}(T(f, g))$ may appear in this combination. This proves 2$)$.

If $\overline{T(f, g)} \notin\langle\bar{f}, \bar{g}\rangle$, then by Corollary 1 we have $\operatorname{deg}_{y} T(x, y) \geq p$ and hence $q(x, y) \neq 0$. By Corollary 1 again, $\overline{s(f, g)} \in\langle\bar{f}, \bar{g}\rangle$; hence $\overline{T(f, g)} \neq \overline{s(f, g)}$. Consequently, by Lemma 6

$$
\operatorname{deg}(T(f, g))=\operatorname{deg}(w(f, g) q(f, g)) \geq \operatorname{deg}(s(f, g)) .
$$

It follows from the definition of $w(x, y)$ in Lemma 4 that

$$
\operatorname{deg}\left(\frac{\partial w}{\partial x}(f, g)\right)=n(s-1), \quad \operatorname{deg}\left(\frac{\partial w}{\partial y}(f, g)\right)=m(p-1) .
$$

Furthermore,

$$
\frac{\partial T}{\partial x}(x, y)=\frac{\partial w}{\partial x}(x, y) q(x, y)+w(x, y) \frac{\partial q}{\partial x}(x, y)+\frac{\partial s}{\partial x}(x, y) .
$$


Easy calculations give

$$
\begin{aligned}
\operatorname{deg}\left(w(f, g) \frac{\partial q}{\partial x}(f, g)\right) & \leq \operatorname{deg}(w(f, g))+\operatorname{deg}(q(f, g))-\operatorname{deg} f, \\
\operatorname{deg}\left(\frac{\partial s}{\partial x}(f, g)\right) & \leq \operatorname{deg}(T(f, g))-\operatorname{deg} f \\
& =\operatorname{deg}(w(f, g))+\operatorname{deg}(q(f, g))-\operatorname{deg} f, \\
\operatorname{deg}\left(\frac{\partial w}{\partial x}(f, g) q(f, g)\right) & =\operatorname{deg}(q(f, g))+n(s-1)=\operatorname{deg}(q(f, g))+m p-n \\
& =\operatorname{deg}(w(f, g))+\operatorname{deg}(q(f, g))-n+(m p-\operatorname{deg}(w(f, g))) \\
& >\operatorname{deg}(w(f, g))+\operatorname{deg}(q(f, g))-n .
\end{aligned}
$$

Therefore,

$$
\operatorname{deg}\left(\frac{\partial T}{\partial x}(f, g)\right)=\operatorname{deg}(q(f, g))+n(s-1)
$$

Similar calculations give the value of $\operatorname{deg}\left(\frac{\partial T}{\partial y}(f, g)\right)$.

To prove 4) we note first that by Lemma 53$), \operatorname{deg}(w(f, g)) \geq N$. Hence by statement 2$)$ of this lemma, $\operatorname{deg}(q(f, g))<n$ and $\operatorname{deg}(s(f, g))<m p$. By Corollary 1 $\overline{q(f, g)} \in\langle\bar{f}, \bar{g}\rangle$. Hence $0 \neq q(x, y)=\lambda \in F$. Now it is easy to see that the polynomial $w_{1}(x, y)=\lambda^{-1} T(x, y)=w(x, y)+\lambda^{-1} s(x, y)$ is a derivative polynomial of the pair $f, g$.

We give two corollaries that will be useful for references.

Corollary 2. If $h \in\langle f, g\rangle \backslash F$ and $\operatorname{deg} h<n$, then $h=\lambda w(f, g), 0 \neq \lambda \in F$, where $w(x, y)$ is a derivative polynomial of the pair $f, g$.

Proof. Put $h=T(f, g)$ and let $\operatorname{deg}_{y} T(x, y)=p q+r, r<p$. If $q=0$, then Corollary 1 gives $\bar{h} \in\langle\bar{f}, \bar{g}\rangle$ and $\operatorname{deg} h \geq n$ or $h \in F$, a contradiction. Hence $q>0$. Inequality (2) gives $\operatorname{deg} h \geq q N+m r$. Consequently, $r=0$. If $q>1$, then by Lemma 2, $\operatorname{deg} h \geq 2 N>n$. Therefore, $q=1$ and $\operatorname{deg}_{y}(T(x, y))=p$. Then Lemma 74) proves the corollary.

Corollary 3. If $w(x, y)$ is a derivative polynomial of the pair $f, g$, then

$$
\begin{aligned}
\operatorname{deg}\left(\frac{\partial w}{\partial x}(f, g)\right) & =n(s-1), \\
\operatorname{deg}\left(\frac{\partial w}{\partial y}(f, g)\right) & =m(p-1) .
\end{aligned}
$$

\section{ReduCtions AND SIMPle AUtomorphisms}

A triple $\theta=\left(f_{1}, f_{2}, f_{3}\right)$ (or simply $\left(f_{1}, f_{2}, f_{3}\right)$ ) of elements of the algebra $A$ below will always denote the automorphism $\theta$ of $A$ such that $\theta\left(x_{i}\right)=f_{i}, 1 \leq i \leq 3$. The number $\operatorname{deg} \theta=\operatorname{deg} f_{1}+\operatorname{deg} f_{2}+\operatorname{deg} f_{3}$ will be called a degree of the automorphism $\theta$.

Recall that an elementary transformation of the triple $\left(f_{1}, f_{2}, f_{3}\right)$ is, by definition, a transformation that changes only one element $f_{i}$ to an element of the form $\alpha f_{i}+g$, where $0 \neq \alpha \in F, g \in\left\langle\left\{f_{j} \mid j \neq i\right\}\right\rangle$. The notation

$$
\left(f_{1}, f_{2}, f_{3}\right) \rightarrow\left(g_{1}, g_{2}, g_{3}\right)
$$

means that the triple $\left(g_{1}, g_{2}, g_{3}\right)$ is obtained from $\left(f_{1}, f_{2}, f_{3}\right)$ by a single elementary transformation. Observe that we do not assume that $\operatorname{deg}\left(g_{1}, g_{2}, g_{3}\right)$ should be 
smaller than $\operatorname{deg} \theta$. An automorphism $\left(f_{1}, f_{2}, f_{3}\right)$ is called tame if there exists a sequence of elementary transformations of the form

$$
\begin{aligned}
\left(x_{1}, x_{2}, x_{3}\right)=\left(f_{1}^{(0)}, f_{2}^{(0)}, f_{3}^{(0)}\right) \rightarrow\left(f_{1}^{(1)}, f_{2}^{(1)}, f_{3}^{(1)}\right) & \rightarrow \ldots \\
& \rightarrow\left(f_{1}^{(n)}, f_{2}^{(n)}, f_{3}^{(n)}\right)=\left(f_{1}, f_{2}, f_{3}\right) .
\end{aligned}
$$

The element $f_{1}$ of the automorphism $\theta=\left(f_{1}, f_{2}, f_{3}\right)$ is called reducible, if there exists $g \in\left\langle f_{2}, f_{3}\right\rangle$ such that $\bar{f}_{1}=\bar{g}$; otherwise it is called unreducible. Put $f_{1}^{\prime}=$ $\alpha\left(f_{1}-g\right)$, where $0 \neq \alpha \in F$; then $\operatorname{deg} f_{1}^{\prime}<\operatorname{deg} f_{1}$ and $\operatorname{deg}\left(f_{1}^{\prime}, f_{2}, f_{3}\right)<\operatorname{deg} \theta$. In this case we will say also that $f_{1}$ is reduced in $\theta$ by the automorphism $\left(f_{1}^{\prime}, f_{2}, f_{3}\right)$. If one of the elements $f_{1}, f_{2}, f_{3}$ of $\theta$ is reducible, then we will say that $\theta$ admits an elementary reduction or simply that $\theta$ is elementary reducible.

Lemma 8. The elementary reducibility of automorphisms of the algebra $A$ is algorithmically recognizable1 1

Proof. Let $\theta=\left(f_{1}, f_{2}, f_{3}\right)$ be an arbitrary automorphism of $A$. We will recognize the reducibility of $f_{3}$. If $\bar{f}_{1}, \bar{f}_{2}$ are algebraically independent, then $f_{3}$ is reducible if and only if $\bar{f}_{3} \in\left\langle\bar{f}_{1}, \bar{f}_{2}\right\rangle$. Since $\bar{f}_{1}, \bar{f}_{2}$ are homogeneous, this question can be solved trivially, even without a reference to the solubility of the occurrence problem [13, 14]. If $\bar{f}_{2} \in\left\langle\bar{f}_{1}\right\rangle$ and $\bar{f}_{2}=\alpha \bar{f}_{1}^{k}$, then the element $f_{3}$ is reducible in $\theta$ if and only if it is reducible in the automorphism $\left(f_{1}, f_{2}-\alpha f_{1}^{k}, f_{3}\right)$. Since $\operatorname{deg}\left(f_{1}, f_{2}-\alpha f_{1}^{k}, f_{3}\right)<$ $\operatorname{deg} \theta$, the statement of the lemma in this case can be proved by induction on $\operatorname{deg} \theta$.

Let now $f_{1}, f_{2}$ be a $*$-reduced pair and $\operatorname{deg} f_{1}<\operatorname{deg} f_{2}$. Assume that there exists a polynomial $G(x, y) \in F[x, y]$ such that $\bar{f}_{3}=\overline{G\left(f_{1}, f_{2}\right)}$. Inequalities (2), (3) gives a bound $k$ for the numbers $\operatorname{deg}_{x}(G(x, y)), \operatorname{deg}_{y}(G(x, y))$. Then $G\left(f_{1}, f_{2}\right)$ is in the space generated by the elements $f_{1}^{i} f_{2}^{j}$, where $i, j \leq k$. The highest homogeneous parts of elements of this space can be described by triangulation.

Now we give an example of a tame automorphism, which does not admit an elementary reduction.

Example 1. Put

$$
\begin{gathered}
h_{1}=x_{1}, \quad h_{2}=x_{2}+x_{1}^{2}, \quad h_{3}=x_{3}+2 x_{1} x_{2}+x_{1}^{3}, \\
g_{1}=6 h_{1}+6 h_{2} h_{3}+h_{3}^{3}, \quad g_{2}=4 h_{2}+h_{3}^{2}, \quad g_{3}=h_{3} .
\end{gathered}
$$

It is easy to show that $\left(h_{1}, h_{2}, h_{3}\right)$ and $\left(g_{1}, g_{2}, g_{3}\right)$ are tame automorphisms of the algebra $A$. Note that $\operatorname{deg} g_{1}=9, \operatorname{deg} g_{2}=6, \operatorname{deg} g_{3}=3$ and $g_{1}, g_{2}$ form a 2-reduced pair. A direct calculation shows that the element

$$
f=g_{1}^{2}-g_{2}^{3}
$$

has degree 8. Hence $\operatorname{deg} f<\operatorname{deg} g_{1}$, and $\bar{f} \notin\left\langle\bar{g}_{1}, \bar{g}_{2}\right\rangle$.

Now we define a tame automorphism $\left(f_{1}, f_{2}, f_{3}\right)$ by putting

$$
f_{1}=g_{1}+g_{3}+f, \quad f_{2}=g_{2}, \quad f_{3}=g_{3}+f .
$$

We have $\operatorname{deg} f_{1}=9, \operatorname{deg} f_{2}=6, \operatorname{deg} f_{3}=8$ and $\operatorname{deg}\left[f_{i}, f_{j}\right]>9,1 \leq i<j \leq 3$. Then, using inequality (2), it is easy to check that the automorphism $\left(f_{1}, f_{2}, f_{3}\right)$ does not admit an elementary reduction.

\footnotetext{
${ }^{1}$ In formulation of algorithmic results, we always assume that the ground field $F$ is constructive.
} 
Proposition 1. Let $\theta=\left(f_{1}, f_{2}, f_{3}\right)$ be an automorphism of $A$ such that $\operatorname{deg} f_{1}=$ $2 n, \operatorname{deg} f_{2}=n s, s \geq 3$ is an odd number, $2 n<\operatorname{deg} f_{3} \leq n s, \bar{f}_{3} \notin\left\langle\bar{f}_{1}, \bar{f}_{2}\right\rangle$. Suppose that there exists $0 \neq \alpha \in F$ such that the elements $g_{1}=f_{1}, g_{2}=f_{2}-\alpha f_{3}$ satisfy the conditions:

i) $g_{1}, g_{2}$ is a 2-reduced pair and $\operatorname{deg} g_{1}=2 n, \operatorname{deg} g_{2}=n s$;

ii) the element $f_{3}$ of the automorphism $\left(g_{1}, g_{2}, f_{3}\right)$ is reduced by an automorphism $\left(g_{1}, g_{2}, g_{3}\right)$ with the condition $\operatorname{deg}\left[g_{1}, g_{3}\right]<n s+\operatorname{deg}\left[g_{1}, g_{2}\right]$.

Then, the following statements are true:

1) $\overline{\left[f_{1}, f_{2}\right]}=\alpha \overline{\left[f_{1}, f_{3}\right]}$;

2) $\operatorname{deg}\left[f_{i}, f_{j}\right]>n s$, where $1 \leq i<j \leq 3$;

3) if $f \in\left\langle f_{i}, f_{j}\right\rangle$, where $1 \leq i<j \leq 3$, then either $\bar{f} \in\left\langle\bar{f}_{i}, \bar{f}_{j}\right\rangle$ or $\operatorname{deg} f>n s$;

4) $\operatorname{deg} f_{1}+\operatorname{deg} f_{3}>n s$.

Proof. We have

$$
g_{3}=\sigma f_{3}+G\left(g_{1}, g_{2}\right), \quad \operatorname{deg} g_{3}<\operatorname{deg} f_{3},
$$

where $0 \neq \sigma \in F, G(x, y) \in F[x, y]$. Hence

$$
\overline{G\left(g_{1}, g_{2}\right)}=-\sigma \bar{f}_{3} .
$$

If $\operatorname{deg} f_{3}=n s$, then $\bar{f}_{2}, \bar{f}_{3}$ are linearly independent, since $\bar{f}_{3} \notin\left\langle\bar{f}_{1}, \bar{f}_{2}\right\rangle$. Therefore $\bar{f}_{2}, \bar{f}_{3}, \bar{g}_{2}$ are mutually linearly independent and $\bar{f}_{3} \notin\left\langle\bar{f}_{1}, \bar{g}_{2}\right\rangle=\left\langle\bar{g}_{1}, \bar{g}_{2}\right\rangle$. If $\operatorname{deg} f_{3}<$ $n s$, then $\bar{f}_{2}=\bar{g}_{2}$ and again we have $\bar{f}_{3} \notin\left\langle\bar{g}_{1}, \bar{g}_{2}\right\rangle$. Put $\operatorname{deg}_{y}(G(x, y))=k=2 q+r$, $0 \leq r \leq 1$. The condition $\bar{f}_{3} \notin\left\langle\bar{g}_{1}, \bar{g}_{2}\right\rangle$ implies, by Corollary 1 and (5), that $q \geq 1$. Then inequality (2) gives $r=0$ and

$$
n s \geq \operatorname{deg}\left(G\left(g_{1}, g_{2}\right)\right)=\operatorname{deg} f_{3} \geq q\left(n s-2 n+\operatorname{deg}\left[g_{1}, g_{2}\right]\right) .
$$

It is easy to deduce from here that if $s>3$, then $q=1, k=2$, and if $s=3$, then $q=1,2, k=2,4$. Besides, these inequalities imply $\operatorname{deg}\left[g_{1}, g_{2}\right] \leq 2 n$ and statement 4) of the proposition.

Applying (11), we get from (4),

$$
\left[g_{1}, g_{3}\right]=\sigma\left[g_{1}, f_{3}\right]+\left[g_{1}, g_{2}\right] \frac{\partial G}{\partial y}\left(g_{1}, g_{2}\right) .
$$

Since $\operatorname{deg}_{y}\left(\frac{\partial G}{\partial y}\right)=k-1$ is an odd number, inequality (2) gives

$$
\operatorname{deg}\left(\left[g_{1}, g_{2}\right] \frac{\partial G}{\partial y}\left(g_{1}, g_{2}\right)\right) \geq \operatorname{deg}\left[g_{1}, g_{2}\right]+n s .
$$

Consequently, by condition ii),

$$
\operatorname{deg}\left[f_{1}, f_{3}\right]=\operatorname{deg}\left[g_{1}, f_{3}\right] \geq \operatorname{deg}\left[g_{1}, g_{2}\right]+n s .
$$

Since $\operatorname{deg}\left[g_{1}, g_{2}\right] \leq 2 n$ and $\alpha \neq 0$, the equality

$$
\left[g_{1}, g_{2}\right]=\left[f_{1}, f_{2}\right]-\alpha\left[f_{1}, f_{3}\right]
$$

gives statement 1 ) of the proposition and

$$
\operatorname{deg}\left[f_{1}, f_{2}\right]=\operatorname{deg}\left[f_{1}, f_{3}\right]>n s .
$$

If $\operatorname{deg} f_{3}=n s$, then, as was remarked earlier, $\bar{f}_{2}, \bar{f}_{3}$ are algebraically independent, and so (see [16])

$$
\operatorname{deg}\left[f_{2}, f_{3}\right]=\operatorname{deg} f_{2}+\operatorname{deg} f_{3}>n s .
$$


If $\operatorname{deg} f_{3}<n s$, then we have

$$
\operatorname{deg}\left[f_{1}, f_{2}\right]+\operatorname{deg} f_{3}<\operatorname{deg}\left[f_{1}, f_{3}\right]+\operatorname{deg} f_{2} .
$$

By Lemma 1

$$
\operatorname{deg}\left[f_{2}, f_{3}\right]+\operatorname{deg} f_{1}=\operatorname{deg}\left[f_{1}, f_{3}\right]+\operatorname{deg} f_{2}
$$

hence

$$
\operatorname{deg}\left[f_{2}, f_{3}\right]=\operatorname{deg}\left[f_{1}, f_{3}\right]+n(s-2)>n s .
$$

Thus statement 2) of the proposition is proved.

To prove 3 ), it suffices by F2) to consider only the case when $\bar{f}_{i}, \bar{f}_{j}$ are algebraically dependent. It is easily seen that $f_{1}, f_{2}$ and $f_{1}, f_{3}$ are $*$-reduced pairs. Suppose that $\bar{f}_{2} \in\left\langle\bar{f}_{3}\right\rangle$. If $\operatorname{deg} f_{2}=\operatorname{deg} f_{3}$ then $\bar{f}_{3} \in\left\langle\bar{f}_{2}\right\rangle$, which contradicts the condition of the proposition. Otherwise $\operatorname{deg} f_{2} \geq 2 \operatorname{deg} f_{3}>\operatorname{deg} f_{1}+\operatorname{deg} f_{3}$, which contradicts 4 ). Consequently, the pair $f_{i}, f_{j}$ is $*$-reduced for every $i \neq j$ and Corollary 1 by Lemma 2.ii) implies 3).

Definition 1. If an automorphism $\theta=\left(f_{1}, f_{2}, f_{3}\right)$ satisfies the conditions of proposition 1, then we will say that $\theta$ admits a reduction of type I, and the automorphism $\left(g_{1}, g_{2}, g_{3}\right)$ will be called a reduction of type I of the automorphism $\theta$, with an active element $f_{3}$.

The automorphism from Example 1 admits a reduction of type I.

Proposition 2. Let $\theta=\left(f_{1}, f_{2}, f_{3}\right)$ be an automorphism of $A$ such that $\operatorname{deg} f_{1}=$ $2 n$, $\operatorname{deg} f_{2}=3 n, \frac{3 n}{2}<\operatorname{deg} f_{3} \leq 2 n$, and $\bar{f}_{1}, \bar{f}_{3}$ are linearly independent. Suppose that there exist $\alpha, \beta \in F$, where $(\alpha, \beta) \neq(0,0)$, such that the elements $g_{1}=f_{1}-\alpha f_{3}$, $g_{2}=f_{2}-\beta f_{3}$ satisfy the conditions:

i) $g_{1}, g_{2}$ is a 2-reduced pair and $\operatorname{deg} g_{1}=2 n$, $\operatorname{deg} g_{2}=3 n$;

ii) the element $f_{3}$ of the automorphism $\left(g_{1}, g_{2}, f_{3}\right)$ is reduced by an automorphism $\left(g_{1}, g_{2}, g_{3}\right)$ with the condition $\operatorname{deg}\left[g_{1}, g_{3}\right]<3 n+\operatorname{deg}\left[g_{1}, g_{2}\right]$.

Then, the following statements are true:

1) $\alpha \in F$ is the solution of the equation $\overline{\left[f_{1}, f_{2}\right]}=\alpha \overline{\left[f_{3}, f_{2}\right]}$, or $\alpha=0$ if it has no solution;

2) $\beta \in F$ is the solution of the equation $\overline{\left[g_{1}, f_{2}\right]}=\beta \overline{\left[g_{1}, f_{3}\right]}$, or $\beta=0$ if it has no solution;

3) $\operatorname{deg}\left[f_{i}, f_{j}\right]>3 n$, where $1 \leq i<j \leq 3$;

4) if $f \in\left\langle f_{i}, f_{j}\right\rangle$, where $1 \leq i<j \leq 3$, then either $\bar{f} \in\left\langle\bar{f}_{i}, \bar{f}_{j}\right\rangle$ or $\operatorname{deg} f>3 n$.

Proof. Consider equalities (4), (5). If $\operatorname{deg} f_{3}<2 n$, then obviously $\overline{G\left(g_{1}, g_{2}\right)} \notin$ $\left\langle\bar{g}_{1}, \bar{g}_{2}\right\rangle$. If $\operatorname{deg} f_{3}=2 n$, then by the condition of the proposition, $\bar{f}_{1}, \bar{f}_{3}$ are linearly independent. Therefore, either $\alpha=0$ and $g_{1}=f_{1}$, or $\alpha \neq 0$ and $\bar{g}_{1}, \bar{f}_{1}, \bar{f}_{3}$ are mutually linearly independent. In any case, $\overline{G\left(g_{1}, g_{2}\right)} \notin\left\langle\bar{g}_{1}, \bar{g}_{2}\right\rangle$. Since $\operatorname{deg} f_{3} \leq 2 n$, then, as in the proof of Proposition 1 inequality (2) gives that $\operatorname{deg}_{y}(G(x, y))=2$, $\operatorname{deg}\left[g_{1}, g_{2}\right] \leq n$. Consequently, Lemma 74$)$ gives that $G(x, y)$ is a derivative polynomial (up to a nonzero scalar factor) of the pair $g_{1}, g_{2}$, and by Corollary 3 ,

$$
\operatorname{deg}\left(\frac{\partial G}{\partial y}\left(g_{1}, g_{2}\right)\right)=3 n
$$

From here, as in the proof of Proposition 1, we get

$$
\operatorname{deg}\left[f_{1}, f_{3}\right]=\operatorname{deg}\left[g_{1}, f_{3}\right]=\operatorname{deg}\left[g_{1}, g_{2}\right]+3 n .
$$


Consider the triple $\left(g_{1}, g_{2}, f_{3}\right)$. By (6) and Lemma 1,

$$
\operatorname{deg}\left[g_{2}, f_{3}\right]+\operatorname{deg} g_{1}=\operatorname{deg}\left[g_{1}, f_{3}\right]+\operatorname{deg} g_{2},
$$

which yields

$$
\operatorname{deg}\left[f_{2}, f_{3}\right]=\operatorname{deg}\left[g_{2}, f_{3}\right]=\operatorname{deg}\left[g_{1}, f_{3}\right]+n .
$$

Furthermore,

$$
\left[f_{1}, f_{2}\right]=\left[g_{1}+\alpha f_{3}, g_{2}+\beta f_{3}\right]=\left[g_{1}, g_{2}\right]+\beta\left[g_{1}, f_{3}\right]+\alpha\left[f_{3}, g_{2}\right] .
$$

Since $\operatorname{deg}\left[g_{1}, g_{2}\right] \leq n$, this implies, by (6) and (7), that $\overline{\left[f_{1}, f_{2}\right]}=\alpha \overline{\left[f_{3}, g_{2}\right]}=\alpha \overline{\left[f_{3}, f_{2}\right]}$ if $\alpha \neq 0$, and $\overline{\left[f_{1}, f_{2}\right]}=\beta \overline{\left[g_{1}, f_{3}\right]}$ if $\alpha=0$. Hence $\operatorname{deg}\left[f_{1}, f_{2}\right]>3 n$, and if $\alpha=0$, then $\left[f_{1}, f_{2}\right],\left[f_{3}, f_{2}\right]$ have different degrees. We have also

$$
\left[g_{1}, f_{2}\right]=\left[g_{1}, g_{2}+\beta f_{3}\right]=\left[g_{1}, g_{2}\right]+\beta\left[g_{1}, f_{3}\right] .
$$

Hence either $\beta \neq 0$ and $\overline{\left[g_{1}, f_{2}\right]}=\beta \overline{\left[g_{1}, f_{3}\right]}$, or the elements $\left[g_{1}, f_{2}\right]=\left[g_{1}, g_{2}\right]$ and $\left[g_{1}, f_{3}\right]$ have different degrees. This proves the statements 1$\left.\left.), 2\right), 3\right)$ of the proposition. Finally, as in the proof of Proposition 1 Corollary 11 and Lemma 2,ii) give 4$)$.

Definition 2. If an automorphism $\theta=\left(f_{1}, f_{2}, f_{3}\right)$ satisfies the conditions of proposition 2 then we will say that $\theta$ admits a reduction of type II, and the automorphism $\left(g_{1}, g_{2}, g_{3}\right)$ will be called a reduction of type II of the automorphism $\theta$, with an active element $f_{3}$.

Proposition 3. Let $\theta=\left(f_{1}, f_{2}, f_{3}\right)$ be an automorphism of $A$ such that $\operatorname{deg} f_{1}=$ $2 n$, and either $\operatorname{deg} f_{2}=3 n, n<\operatorname{deg} f_{3} \leq \frac{3 n}{2}$, or $\frac{5 n}{2}<\operatorname{deg} f_{2} \leq 3 n, \operatorname{deg} f_{3}=\frac{3 n}{2}$. Suppose that there exist $\alpha, \beta, \gamma \in F$ such that the elements $g_{1}=f_{1}-\beta f_{3}, g_{2}=$ $f_{2}-\gamma f_{3}-\alpha f_{3}^{2}$ satisfy the conditions:

i) $g_{1}, g_{2}$ is a 2-reduced pair and $\operatorname{deg} g_{1}=2 n, \operatorname{deg} g_{2}=3 n$;

ii) there exists an element $g_{3}$ of the form

$$
g_{3}=\sigma f_{3}+g,
$$

where $0 \neq \sigma \in F, g \in\left\langle g_{1}, g_{2}\right\rangle \backslash F$, such that $\operatorname{deg} g_{3} \leq \frac{3 n}{2}, \operatorname{deg}\left[g_{1}, g_{3}\right]<$ $3 n+\operatorname{deg}\left[g_{1}, g_{2}\right]$.

Then, the following statements are true:

1) $\alpha \in F$ is the solution of the equation $\overline{\left[f_{1}, f_{2}\right]}=2 \alpha \overline{\left[f_{1}, f_{3}\right]} \bar{f}_{3}$, or $\alpha=0$ if it has no solution;

2) $\beta \in F$ is the solution of the equation $\overline{\left[f_{2}-\alpha f_{3}^{2}, f_{1}\right]}=\beta \overline{\left[f_{2}, f_{3}\right]}$, or $\beta=0$ if it has no solution;

3) $\gamma \in F$ is the solution of the equation $\overline{\left[g_{1}, f_{2}-\alpha f_{3}^{2}\right]}=\gamma \overline{\left[g_{1}, f_{3}\right]}$, or $\gamma=0$ if it has no solution;

4) $\operatorname{deg}\left[f_{1}, f_{3}\right], \operatorname{deg}\left[f_{2}, f_{3}\right]>3 n$;

5) if $(\alpha, \beta, \gamma) \neq(0,0,0)$, then $\operatorname{deg}\left[f_{1}, f_{2}\right]>3 n$; otherwise, $\operatorname{deg}\left[f_{1}, f_{2}\right]=$ $\operatorname{deg}\left[g_{1}, g_{2}\right] \leq \frac{n}{2}$

6) if $\bar{g}_{2}=-\alpha \bar{f}_{3}^{2}$, then $\frac{5 n}{2}+\operatorname{deg}\left[g_{1}, g_{2}\right] \leq \operatorname{deg} f_{2}<3 n$; otherwise, $\operatorname{deg} f_{2}=3 n$.

Proof. Since $\operatorname{deg} f_{3}, \operatorname{deg} g_{3} \leq \frac{3 n}{2}$, condition ii) yields that $\operatorname{deg} g \leq \frac{3 n}{2}$. Then, by Corollary 2, $g=\lambda w\left(g_{1}, g_{2}\right)$, where $w(x, y)$ is a derivative polynomial of the $g_{1}, g_{2}$. 
Inequality (2) gives also $\operatorname{deg}\left[g_{1}, g_{2}\right] \leq \frac{n}{2}$. As in the proof of Propositions 10 2, we obtain also (6), (7), which yields 4). Besides, we have

$$
\left[f_{1}, f_{2}\right]=\left[g_{1}, g_{2}\right]+\gamma\left[g_{1}, f_{3}\right]+\beta\left[f_{3}, g_{2}\right]+2 \alpha\left[g_{1}, f_{3}\right] f_{3} .
$$

Since $\operatorname{deg} f_{3}>n$, this equality yields statements 1 ), 5) of the proposition. If $\bar{g}_{2}=-\alpha \bar{f}_{3}^{2}$, then $\alpha \neq 0, \operatorname{deg} f_{3}=\frac{3 n}{2}, \operatorname{deg} f_{2}<3 n$, and

$$
\operatorname{deg}\left[f_{1}, f_{2}\right]=\operatorname{deg}\left[g_{1}, f_{3}\right]+\operatorname{deg} f_{3}=\operatorname{deg}\left[g_{1}, g_{2}\right]+3 n+\frac{3 n}{2} .
$$

Consequently, $\operatorname{deg} f_{2} \geq \operatorname{deg}\left[g_{1}, g_{2}\right]+\frac{5 n}{2}$, which proves 6$)$. We have also

$$
\begin{aligned}
& {\left[f_{2}-\alpha f_{3}^{2}, f_{1}\right]=\left[g_{2}, g_{1}\right]+\gamma\left[f_{3}, g_{1}\right]+\beta\left[g_{2}, f_{3}\right],} \\
& {\left[g_{1}, f_{2}-\alpha f_{3}^{2}\right]=\left[g_{1}, g_{2}\right]+\gamma\left[g_{1}, f_{3}\right] .}
\end{aligned}
$$

These equalities imply statements 2), 3) of the proposition.

Definition 3. If an automorphism $\theta=\left(f_{1}, f_{2}, f_{3}\right)$ satisfies the conditions of Proposition 3, and $(\alpha, \beta, \gamma) \neq(0,0,0), \operatorname{deg} g_{3}<n+\operatorname{deg}\left[g_{1}, g_{2}\right]$, then we will say that $\theta$ admits a reduction of type III, and the automorphism $\left(g_{1}, g_{2}, g_{3}\right)$ will be called a reduction of type III of the automorphism $\theta$, with an active element $f_{3}$.

Corollary 4. In the conditions of Proposition [3, if the automorphism $\theta=$ $\left(f_{1}, f_{2}, f_{3}\right)$ admits a reduction of type III, then $\operatorname{deg}\left(g_{1}, g_{2}, g_{3}\right)<\operatorname{deg} \theta$.

Proof. By Definition [3, we have

$$
\operatorname{deg}\left(g_{1}, g_{2}, g_{3}\right)<6 n+\operatorname{deg}\left[g_{1}, g_{2}\right] .
$$

Hence it is sufficient to prove that

$$
\operatorname{deg} \theta \geq 6 n+\operatorname{deg}\left[g_{1}, g_{2}\right] .
$$

It follows from (6) that $\operatorname{deg} f_{3} \geq n+\operatorname{deg}\left[g_{1}, g_{2}\right]$. If $\bar{g}_{2} \neq-\alpha \bar{f}_{3}^{2}$, then Proposition 36 ) gives $\operatorname{deg} f_{2}=3 n$, which proves (8). If $\bar{g}_{2}=-\alpha \bar{f}_{3}^{2}$, then, as shown above, $\operatorname{deg} f_{3}=$ $\frac{3 n}{2}$ and by Proposition 3.6), $\operatorname{deg} f_{2} \geq \frac{5 n}{2}+\operatorname{deg}\left[g_{1}, g_{2}\right]$, which also gives (8).

Definition 4. If an automorphism $\theta=\left(f_{1}, f_{2}, f_{3}\right)$ satisfies the conditions of Proposition 3 and there exists $0 \neq \mu \in F$ such that $\operatorname{deg}\left(g_{2}-\mu g_{3}^{2}\right) \leq 2 n$, then we will say that $\theta$ admits a reduction of type IV, and the automorphism $\left(g_{1}, g_{2}-\mu g_{3}^{2}, g_{3}\right)$ will be called a reduction of type IV of the automorphism $\theta$, with an active element $f_{3}$. In this case we will also call the automorphism $\left(g_{1}, g_{2}, g_{3}\right)$ a predreduction of type IV of $\theta$.

Corollary 5. If an automorphism $\theta=\left(f_{1}, f_{2}, f_{3}\right)$ satisfies all the conditions of Proposition 3 and Definition 4 then $\operatorname{deg}\left(g_{1}, g_{2}-\mu g_{3}^{2}, g_{3}\right)<\operatorname{deg} \theta$.

Proof. Since $\operatorname{deg}\left(g_{2}-\mu g_{3}^{2}\right) \leq 2 n$, then $\bar{g}_{2}=\mu{\overline{g_{3}}}^{2}$ and $\operatorname{deg} g_{3}=\frac{3 n}{2}$. Consequently, $\operatorname{deg}\left(g_{1}, g_{2}-\mu g_{3}^{2}, g_{3}\right) \leq \frac{11 n}{2}$. Inequality (8) completes the proof.

Reductions of types I-IV, together with elementary reductions, permit us to introduce an auxiliary notion of a simple automorphism.

Definition 5. By induction on degree, we will define simple automorphisms of the algebra $A$ as follows.

1) All the automorphisms of degree 3 are simple.

2) Suppose that the simple automorphisms of degree $<n$ are already defined. 
3) An automorphism $\theta$ of degree $n>3$ is called simple if there exists a simple automorphism of degree $<n$ that is either an elementary reduction or a reduction of type I-IV of $\theta$.

Evidently, any simple automorphism is tame. Our principal goal is to prove the converse statement, that every tame automorphism is simple. We will do it in the next section.

Remark 1. If $\left(f_{1}, f_{2}, f_{3}\right)$ is a simple automorphism, then the automorphisms $\left(f_{2}, f_{1}, f_{3}\right)$ and $\left(\alpha f_{1}+\beta, f_{2}, f_{3}\right)$, where $\alpha, \beta \in F, \alpha \neq 0$, are simple as well.

Really, if $\left(g_{1}, g_{2}, g_{3}\right)$ is an elementary reduction or a reduction of type I-IV of $\left(f_{1}, f_{2}, f_{3}\right)$, then $\left(g_{2}, g_{1}, g_{3}\right)$ is a reduction of the same type for $\left(f_{2}, f_{1}, f_{3}\right)$. It is also clear that we can always choose $\alpha_{i}, \beta_{i} \in F$ such that $\left(\alpha_{1} g_{1}+\beta_{1}, \alpha_{2} g_{2}+\beta_{2}, \alpha_{3} g_{3}+\beta_{3}\right)$ becomes a corresponding reduction for $\left(\alpha f_{1}+\beta, f_{2}, f_{3}\right)$.

For convenience of terminology, we introduce also

Definition 6. An element $f_{i}, i=1,2,3$, of the automorphism $\theta=\left(f_{1}, f_{2}, f_{3}\right)$ is called simple reducible if it is reduced by a simple automorphism.

\section{A characterization of tame aUtomorphisms}

This section is devoted to the proof of our main result.

Theorem 1. Every tame automorphism of the algebra $A$ is simple.

The plan of the proof. Assume that the statement of the theorem is not true. Then there exist tame automorphisms $\theta=\left(f_{1}, f_{2}, f_{3}\right), \tau$ of $A$ such that $\theta$ is simple, $\tau$ is not simple, and

$$
\theta=\left(f_{1}, f_{2}, f_{3}\right) \rightarrow \tau .
$$

In the set of all pairs of automorphisms with this property we choose and fix a pair $\theta, \tau$ with the minimal $\operatorname{deg} \theta$.

In order to obtain a contradiction, it is enough to prove that $\tau$ is simple. The proof will consist of analysis of the cases, when $\theta$ admits an elementary reduction or a reduction of type I-IV to a simple automorphism of lower degree. If $\theta$ admits a reduction of type I-IV, then it will be convenient for us to fix the reduction of $\theta$ and consider one of the following variants for $\tau$ :

$$
\begin{aligned}
& \tau=\left(f, f_{2}, f_{3}\right), \quad f=f_{1}+a, \quad a \in\left\langle f_{2}, f_{3}\right\rangle, \quad \operatorname{deg} a \leq \operatorname{deg} f_{1}, \\
& \tau=\left(f_{1}, f, f_{3}\right), \quad f=f_{2}+a, \quad a \in\left\langle f_{1}, f_{3}\right\rangle, \quad \operatorname{deg} a \leq \operatorname{deg} f_{2}, \\
& \tau=\left(f_{1}, f_{2}, f\right), \quad f=f_{3}+a, \quad a \in\left\langle f_{1}, f_{2}\right\rangle, \quad \operatorname{deg} a \leq \operatorname{deg} f_{3} .
\end{aligned}
$$

Here, the restriction on $\operatorname{deg} a$ is imposed in order to exclude the trivial case when $\theta$ is an elementary reduction of $\tau$. In the case when $\theta$ admits an elementary reduction, we will assume that $\tau$ has form (11). The proof of the theorem will be completed by Lemmas 917 and by Propositions 4 , 5 .

The following evident statement is formulated for convenience of references.

Lemma 9. Let $\phi$ be a simple automorphism of $A$ such that $\operatorname{deg} \phi<\operatorname{deg} \theta$. If $\phi \rightarrow \psi$, then $\psi$ is simple too.

The proof follows immediately from the minimality condition for $\operatorname{deg} \theta$. 
Corollary 6. Suppose that there exists a sequence of automorphisms

$$
\phi_{0} \rightarrow \phi_{1} \rightarrow \ldots \rightarrow \phi_{k-1} \rightarrow \phi_{k}
$$

of the algebra $A$ such that $\operatorname{deg} \phi_{i}<\operatorname{deg} \theta$ for $0 \leq i \leq k-1$, and $\phi_{0}$ is a simple automorphism. Then $\phi_{k}$ is also simple.

Lemma 10. If $\theta$ admits a reduction of type $I$, then $\tau$ is simple.

Proof. We adopt all the conditions and notation of Proposition 1 and by the definition of reduction we have that $\left(g_{1}, g_{2}, g_{3}\right)$ is simple. Without loss of generality, we can also put

$$
g_{3}=f_{3}+g, \quad g \in\left\langle g_{1}, g_{2}\right\rangle, \operatorname{deg} g_{3}<\operatorname{deg} f_{3} .
$$

If $\tau$ is of form (9), then by Proposition[1,3) we have $\bar{a} \in\left\langle\bar{f}_{2}, \bar{f}_{3}\right\rangle$. Since $\operatorname{deg} a \leq$ $\operatorname{deg} f_{1}<\min \left\{\operatorname{deg} f_{2}, \operatorname{deg} f_{3}\right\}$, this implies $a \in F$. Hence $\tau$ is simple by Remark 1

Assume that $\tau$ has form (10). By statements 3) and 4) of Proposition 1 we have

$$
\bar{a}=\beta \bar{f}_{3}+\gamma\left(\bar{f}_{1}\right)^{k}, \beta, \gamma \in F, 2 n k \leq \operatorname{deg} f_{2} .
$$

Consider $a_{1}=a-\beta f_{3}-\gamma\left(f_{1}\right)^{k}$. Then again $a_{1} \in\left\langle f_{1}, f_{3}\right\rangle$ and $\operatorname{deg} a_{1}<\operatorname{deg} a$; hence $\bar{a}_{1} \in\left\langle\bar{f}_{1}\right\rangle$, and it is easy to see that $a_{1} \in\left\langle f_{1}\right\rangle$. Thus we have

$$
a=\beta f_{3}+T\left(f_{1}\right), \quad \operatorname{deg}\left(T\left(f_{1}\right)\right) \leq \operatorname{deg} f_{2}, \quad f=f_{2}+\beta f_{3}+T\left(f_{1}\right) .
$$

Since $\operatorname{deg} f_{1}=2 n, \operatorname{deg} f_{2}=s n$, where $s$ is odd, $\operatorname{deg}\left(T\left(f_{1}\right)\right)<\operatorname{deg} f_{2}$. Furthermore, $\operatorname{deg} f$ can be less than $\operatorname{deg} f_{2}$ only if $\bar{f}_{2}=-\beta \bar{f}_{3}$. But $\bar{f}_{3} \notin\left\langle\bar{f}_{1}, \bar{f}_{2}\right\rangle$; hence $\operatorname{deg} f=$ $\operatorname{deg} f_{2}$. Put

$$
g_{2}^{\prime}=f-(\alpha+\beta) f_{3}=\left(f_{2}-\alpha f_{3}\right)+T\left(f_{1}\right)=g_{2}+T\left(g_{1}\right) .
$$

Then $\overline{g_{2}^{\prime}}=\bar{g}_{2}$, and $g \in\left\langle g_{1}, g_{2}\right\rangle=\left\langle g_{1}, g_{2}^{\prime}\right\rangle$. If $\alpha+\beta \neq 0$, then (12) implies that $\left(g_{1}, g_{2}^{\prime}, g_{3}\right)$ is a reduction of type I of $\tau$. If $\alpha+\beta=0$, then $\tau=\left(g_{1}, g_{2}^{\prime}, f_{3}\right)$ and the element $f_{3}$ is reduced in $\tau$ by $\left(g_{1}, g_{2}^{\prime}, g_{3}\right)$. It remains to note that, by Lemma 9 $\left(g_{1}, g_{2}^{\prime}, g_{3}\right)$ is a simple automorphism.

Now consider the case when $\tau$ has form (11). Proposition 1 gives, as before,

$$
a=\beta f_{2}+T\left(f_{1}\right), \quad \operatorname{deg}\left(T\left(f_{1}\right)\right) \leq \operatorname{deg} f_{3}, \quad f=f_{3}+\beta f_{2}+T\left(f_{1}\right),
$$

and $\beta \neq 0$ is possible only if $\operatorname{deg} f_{3}=\operatorname{deg} f_{2}$. Consider 3 cases:

1) $\beta=0,2) \beta(1+\alpha \beta) \neq 0,3) 1+\alpha \beta=0$.

In case 1) we put

$$
g_{2}^{\prime}=f_{2}-\alpha f=g_{2}-\alpha T\left(g_{1}\right) .
$$

Since $\operatorname{deg} g_{1} \backslash \operatorname{deg} g_{2}$, the equality $\operatorname{deg}\left(T\left(g_{1}\right)\right)=\operatorname{deg} g_{2}$ is impossible, $\overline{g_{2}^{\prime}}=\overline{g_{2}}$. By Lemma 9, the automorphism $\phi=\left(g_{1}, g_{2}^{\prime}, g_{3}\right)$ is simple again. Since

$$
f=f_{3}+T\left(f_{1}\right)=g_{3}-g+T\left(g_{1}\right), \quad-g+T\left(g_{1}\right) \in\left\langle g_{1}, g_{2}\right\rangle=\left\langle g_{1}, g_{2}^{\prime}\right\rangle,
$$

$\phi$ is a reduction of type $\mathrm{I}$ of $\tau$.

In case 2) we put

$$
g_{2}^{\prime}=f_{2}-\frac{\alpha}{1+\alpha \beta} f=\frac{1}{1+\alpha \beta} g_{2}-\frac{\alpha}{1+\alpha \beta} T\left(g_{1}\right) .
$$

A direct calculation gives

$$
f=(1+\alpha \beta) g_{3}+g^{\prime}
$$


where

$$
g^{\prime}=-(1+\alpha \beta) g+\beta(1+\alpha \beta) g_{2}^{\prime}+(1+\alpha \beta) T\left(g_{1}\right), \quad g^{\prime} \in\left\langle g_{1}, g_{2}\right\rangle=\left\langle g_{1}, g_{2}^{\prime}\right\rangle .
$$

Since $\overline{g_{2}^{\prime}}=\frac{1}{1+\alpha \beta} \overline{g_{2}}$, then $\left(g_{1}, g_{2}^{\prime}, g_{3}\right)$ is a reduction of type I of $\tau$.

In case 3) we have

$$
\begin{aligned}
f & =\beta\left(f_{2}-\alpha f_{3}\right)+T\left(f_{1}\right)=\beta g_{2}+T\left(g_{1}\right), \\
f_{2} & =\alpha g_{3}+\left(g_{2}-\alpha g\right), g_{2}-\alpha g \in\left\langle g_{1}, g_{2}\right\rangle=\left\langle g_{1}, f\right\rangle .
\end{aligned}
$$

Therefore, $\bar{f}=\beta \overline{g_{2}}$, and it is easy to check that $f_{2}$ is reduced in $\tau=\left(g_{1}, f_{2}, f\right)$ by $\left(g_{1}, g_{3}, f\right)$. By Remark $1\left(g_{1}, g_{3}, g_{2}\right)$ is simple. Thus, by Lemma 9 , $\left(g_{1}, g_{3}, f\right)$ is simple too.

Lemma 11. If $\theta$ admits a reduction of type II, then $\tau$ is simple.

Proof. We adopt all the conditions and notation of Proposition 2, as well as equality (12). If $\tau$ has form (9), then Proposition 214) and the condition $\operatorname{deg} a \leq \operatorname{deg} f_{1}$ give $a=\gamma f_{3}+\lambda$. By Remark 1 we may assume that $\lambda=0$. So

$$
\begin{array}{r}
a=\gamma f_{3}, \quad f=f_{1}+\gamma f_{3}, \\
f-(\gamma+\alpha) f_{3}=g_{1}, \quad f_{2}-\beta f_{3}=g_{2} .
\end{array}
$$

If $(\gamma+\alpha, \beta) \neq(0,0)$, then it is easily checked that $\left(g_{1}, g_{2}, g_{3}\right)$ is a reduction of type II of $\tau$. Otherwise, the element $f_{3}$ is reduced in the automorphism $\tau=\left(g_{1}, g_{2}, f_{3}\right)$ by $\left(g_{1}, g_{2}, g_{3}\right)$.

If $\tau$ has form (10), then by Proposition 24) (and Remark 1) we get

$$
a=\gamma f_{3}+\delta f_{1}, \quad f=f_{2}+\gamma f_{3}+\delta f_{1} .
$$

Furthermore,

$$
f-(\gamma+\beta+\delta \alpha) f_{3}=g_{2}+\delta g_{1}=g_{2}^{\prime}, \quad f_{1}-\alpha f_{3}=g_{1} .
$$

If $(\alpha, \gamma+\beta+\delta \alpha) \neq(0,0)$, then $\left(g_{1}, g_{2}^{\prime}, g_{3}\right)$ is a reduction of type II of $\tau$. Otherwise, $\left(g_{1}, g_{2}^{\prime}, g_{3}\right)$ reduces the element $f_{3}$ of $\tau=\left(g_{1}, g_{2}^{\prime}, f_{3}\right)$.

Assume that $\tau$ has form (11). Proposition 2 and Remark 1 give

$$
a=\gamma f_{1}, \quad f=f_{3}+\gamma f_{1},
$$

and $\gamma \neq 0$ is possible only if $\operatorname{deg} f_{1}=\operatorname{deg} f_{3}$. Since $\bar{f}_{1}, \bar{f}_{3}$ are linearly independent, $\operatorname{deg} f=\operatorname{deg} f_{3}$.

If $1+\alpha \gamma \neq 0$, a direct calculation gives

$$
\begin{aligned}
f=f_{3}+\gamma f_{1} & =(1+\alpha \gamma) g_{3}+\left(\gamma g_{1}-(1+\alpha \gamma) g\right), \\
f_{1}-\frac{\alpha}{1+\alpha \gamma} f & =\frac{1}{1+\alpha \gamma} g_{1}=g_{1}^{\prime}, \\
f_{2}-\frac{\beta}{1+\alpha \gamma} f & =g_{2}-\frac{\beta \gamma}{1+\alpha \gamma} g_{1}=g_{2}^{\prime} .
\end{aligned}
$$

Since $\left\langle g_{1}, g_{2}\right\rangle=\left\langle g_{1}^{\prime}, g_{2}^{\prime}\right\rangle$, it is easy to check that $\left(g_{1}^{\prime}, g_{2}^{\prime}, g_{3}\right)$ is a reduction of type II of $\tau$. By Corollary 6 , the automorphism $\left(g_{1}^{\prime}, g_{2}^{\prime}, g_{3}\right)$ is simple.

If $1+\alpha \gamma=0$, then

$$
\begin{array}{r}
f=\gamma g_{1}, f_{1}=\alpha g_{3}+\left(g_{1}-\alpha g\right), \\
f_{2}-\frac{\beta}{\alpha} f_{1}=g_{2}-\frac{\beta}{\alpha} g_{1}=g_{2}^{\prime} .
\end{array}
$$


If $\beta=0$, then the element $f_{1}$ is reduced in $\tau=\left(f_{1}, g_{2}^{\prime}, \gamma g_{1}\right)$ by $\left(g_{3}, g_{2}^{\prime}, \gamma g_{1}\right)$. Otherwise, it is easily checked that $\left(g_{3}, g_{2}^{\prime}, \gamma g_{1}\right)$ is a reduction of type II of $\tau$ with an active element $f_{1}$.

Lemma 12. If $\theta$ admits a reduction of type III or IV, then $\tau$ is simple.

Proof. We adopt the conditions and notation of Proposition 3 Assume that $\tau$ has form (9). Let us show that in this case $a=\delta_{1} f_{3}$.

Evidently, it suffices to prove that, for any $b \in\left\langle f_{2}, f_{3}\right\rangle$ with $\operatorname{deg} b \leq \operatorname{deg} f_{1}=$ $2 n, \bar{b} \in\left\langle\bar{f}_{3}\right\rangle$ holds. Note that $\operatorname{deg} f_{2}>\frac{5 n}{2}>\operatorname{deg} b$. By F2) we may assume, without loss of generality, that $\bar{f}_{2}, \bar{f}_{3}$ are algebraically dependent. If $\bar{f}_{2} \notin\left\langle\bar{f}_{3}\right\rangle$, then the pair $f_{2}, f_{3}$ is $*$-reduced, and the statement holds by Proposition 34 ), Corollary 1, and Lemma 2.ii). Otherwise $\operatorname{deg} f_{3}=\frac{3 n}{2}, \operatorname{deg} f_{2}=3 n, \bar{f}_{2}=\lambda\left(\bar{f}_{3}\right)^{2}$, where $0 \neq \lambda \in F$. Since $\operatorname{deg} g_{2}=3 n$, then $\bar{f}_{2} \neq \alpha \bar{f}_{3}^{2}$, i.e., $\lambda \neq \alpha$. Consider $f_{2}^{\prime}=f_{2}-\lambda f_{3}^{2}=g_{2}+\gamma f_{3}+(\alpha-\lambda) f_{3}^{2}$. Then

$$
\left[f_{1}, f_{2}^{\prime}\right]=\left[g_{1}, g_{2}\right]+\gamma\left[g_{1}, f_{3}\right]+\beta\left[f_{3}, g_{2}\right]+2(\alpha-\lambda)\left[g_{1}, f_{3}\right] f_{3} \text {. }
$$

Since $(\alpha-\lambda) \neq 0$, we have, as in the proof of Proposition 3, that $\operatorname{deg}\left[f_{1}, f_{2}^{\prime}\right]=$ $\operatorname{deg}\left[g_{1}, g_{2}\right]+\frac{9 n}{2}$, which yields $\left.\operatorname{deg} f_{2}^{\prime}\right\rangle \frac{5 n}{2}$. Note that $\left\langle f_{2}^{\prime}, f_{3}\right\rangle=\left\langle f_{2}, f_{3}\right\rangle$. If $\bar{f}_{2}^{\prime}, \bar{f}_{3}$ are algebraically independent, then by F2) we get $\bar{b} \in\left\langle\bar{f}_{2}^{\prime}, \bar{f}_{3}\right\rangle$. Otherwise, $f_{2}^{\prime}, f_{3}$ form a *-reduced pair, and since $\operatorname{deg}\left[f_{2}^{\prime}, f_{3}\right]=\operatorname{deg}\left[f_{2}, f_{3}\right]>3 n$, we have again $\bar{b} \in\left\langle\bar{f}_{2}^{\prime}, \bar{f}_{3}\right\rangle$ by Corollary 1 and Lemma 2 ii). But $\operatorname{deg} f_{2}^{\prime}>\frac{5 n}{2}>\operatorname{deg} b$, and so $\bar{b} \in\left\langle\bar{f}_{3}\right\rangle$.

Thus $a=\delta_{1} f_{3}$, and so

$$
f=f_{1}+\delta_{1} f_{3}=g_{1}+\left(\beta+\delta_{1}\right) f_{3}, \quad f_{2}=g_{2}+\gamma f_{3}+\alpha f_{3}^{2} .
$$

Since $f_{3}$ is preserved in the structure of $\tau=\left(f, f_{2}, f_{3}\right)$, it is easily checked that if $\theta$ admits a reduction of type IV, then $\left(g_{1}, g_{2}, g_{3}\right)$ is a predreduction of type IV of $\tau$. Suppose that $\theta$ admits a reduction of type III. If $\left(\alpha, \beta+\delta_{1}, \gamma\right) \neq(0,0,0)$, then $\left(g_{1}, g_{2}, g_{3}\right)$ is a reduction of type III of $\tau$. Otherwise, since $\operatorname{deg} g_{3}<\operatorname{deg} f_{3}$ (see the proof of Corollary (4), the element $f_{3}$ is reduced in $\tau=\left(g_{1}, g_{2}, f_{3}\right)$ by $\left(g_{1}, g_{2}, g_{3}\right)$.

Suppose that $\tau$ has form (10). Then, by Proposition 34) and Corollary 1 we have $\bar{a} \in\left\langle\bar{f}_{1}, \bar{f}_{3}\right\rangle$. Note that $\operatorname{deg} a \leq \operatorname{deg} f_{2} \leq 3 n$ and $\operatorname{deg}\left(f_{1}^{2}\right), \operatorname{deg}\left(f_{1} f_{3}\right), \operatorname{deg}\left(f_{3}^{3}\right)>3 n$. So

$$
a=\delta_{1} f_{3}^{2}+\sigma_{1} f_{3}+\mu_{1} f_{1},
$$

and $\delta_{1} \neq 0$ is possible only if $\operatorname{deg} f_{2} \geq 2 \operatorname{deg} f_{3}$. Therefore,

$$
f_{1}=g_{1}+\beta f_{3}, \quad f=g_{2}+\mu_{1} g_{1}+\left(\alpha+\delta_{1}\right) f_{3}^{2}+\left(\gamma+\sigma_{1}+\mu_{1} \beta\right) f_{3} .
$$

Hence, if $\operatorname{deg} f \neq 3 n$, then $\overline{g_{2}}+\left(\alpha+\delta_{1}\right){\overline{f_{3}}}^{2}=0$, i.e., $\alpha+\delta_{1} \neq 0, \operatorname{deg} f_{3}=\frac{3 n}{2}$. By Proposition 3 ,

$$
\operatorname{deg}\left[f_{3}, g_{1}\right]=\operatorname{deg}\left[f_{3}, f_{1}\right]>3 n
$$

Since

$$
\left[f, g_{1}\right]=\left[g_{2}, g_{1}\right]+2\left(\alpha+\delta_{1}\right)\left[f_{3}, g_{1}\right] f_{3}+\left(\gamma+\sigma_{1}+\mu_{1} \beta\right)\left[f_{3}, g_{1}\right]
$$

then

$$
\operatorname{deg}\left[f, g_{1}\right]>3 n+\frac{3 n}{2}=\frac{9 n}{2} .
$$

Consequently, $\operatorname{deg} f>\frac{5 n}{2}$. Now it is easy to show, that if $\theta$ admits a reduction of type IV, then $\left(g_{1}, g_{2}+\mu_{1} g_{1}, g_{3}\right)$ is a predreduction of type IV of $\tau$. Suppose now that $\theta$ admits a reduction of type III. If $\left(\alpha+\delta_{1}, \beta, \gamma+\sigma_{1}+\mu_{1} \beta\right) \neq 0$, then 
$\left(g_{1}, g_{2}+\mu_{1} g_{1}, g_{3}\right)$ is a reduction of type III of $\tau$. Otherwise, the element $f_{3}$ is reduced in $\tau=\left(g_{1}, g_{2}+\mu_{1} g_{1}, f_{3}\right)$ by $\left(g_{1}, g_{2}+\mu_{1} g_{1}, g_{3}\right)$.

Assume that $\tau$ has form (11). If $(\alpha, \beta, \gamma) \neq(0,0,0)$, then Proposition 3 yields $a \in F$. If $\alpha=\beta=\gamma=0$, then $\theta$ admits a reduction of type IV. We have

$$
f_{1}=g_{1}, \quad f_{2}=g_{2}, \quad f_{3}=\frac{1}{\sigma} g_{3}-\frac{1}{\sigma} g,
$$

where $g \in\left\langle g_{1}, g_{2}\right\rangle \backslash F$. Since $\operatorname{deg} a \leq \operatorname{deg} f_{3} \leq \frac{3 n}{2}$, we have

$$
f=\frac{1}{\sigma} g_{3}+c,
$$

where $c=-\frac{1}{\sigma} g+a \in\left\langle g_{1}, g_{2}\right\rangle$ and $\operatorname{deg} c \leq \frac{3 n}{2}$. If $c \notin F$, then $\left(g_{1}, g_{2}, g_{3}\right)$ is a predreduction of type IV of $\tau$. Otherwise, by Remark 1 we can take $\tau=\left(g_{1}, g_{2}, \frac{1}{\sigma} g_{3}\right)$, and the element $g_{2}$ is reduced in $\tau$ by $\left(g_{1}, g_{2}-\mu g_{3}^{2}, \frac{1}{\sigma} g_{3}\right)$.

It remains now to consider the principal case, when $\theta$ admits an elementary reduction. It follows from Lemma 9 that if $\phi$ is simple and $\operatorname{deg} \phi<\operatorname{deg} \theta$, then every reducible element of $\phi$ is simple reducible. But one should carefully distinguish these notions if $\operatorname{deg} \phi \geq \operatorname{deg} \theta$.

Lemma 13. Let $\phi=\left(g_{1}, g_{2}, g_{3}\right)$ be a simple automorphism and $\operatorname{deg} \phi \leq \operatorname{deg} \theta$. If $g_{1}$ is a simple reducible element of $\phi$, then every elementary transformation $\psi$ of $\phi$ changing only $g_{1}$ gives a simple automorphism.

Proof. Assume that the element $g_{1}$ is reduced in $\phi$ by a simple automorphism $\phi^{\prime}=\left(h_{1}, g_{2}, g_{3}\right)$. Then $\operatorname{deg} \phi^{\prime}<\operatorname{deg} \theta$ and $h_{1}=\alpha g_{1}+g$, where $0 \neq \alpha \in F$, $g \in\left\langle g_{2}, g_{3}\right\rangle$. Put $\psi=\left(\beta g_{1}+T\left(g_{2}, g_{3}\right), g_{2}, g_{3}\right)$. Then

$$
\beta g_{1}+T\left(g_{2}, g_{3}\right)=\frac{\beta}{\alpha} h_{1}+g^{\prime}, \quad g^{\prime} \in\left\langle g_{2}, g_{3}\right\rangle .
$$

Hence $\phi^{\prime} \rightarrow \psi$ and Lemma 9 completes the proof.

In the sequel we will assume that $\tau$ has form (11) and $\theta$ admits an elementary reduction. Then Lemma 13 gives

Corollary 7. If $f_{3}$ is a simple reducible element of $\theta$, then $\tau$ is a simple automorphism.

In the remainder of this section we will assume that $f_{3}$ is not a simple reducible element of $\theta$. Then either $f_{1}$ or $f_{2}$ are simple reducible. For definiteness, we assume that $f_{2}$ is reduced in $\theta$ by a simple automorphism $\phi=\left(f_{1}, g_{2}, f_{3}\right)$, where

$$
g_{2}=f_{2}+b, \quad b \in\left\langle f_{1}, f_{3}\right\rangle, \quad \operatorname{deg} g_{2}<\operatorname{deg} f_{2} .
$$

Lemma 14. The automorphism $\tau$ is simple if one of the following conditions is satisfied:

1) $\bar{f}_{2} \in\left\langle\bar{f}_{1}\right\rangle$;

2) $\bar{f}_{3} \in\left\langle\bar{f}_{1}\right\rangle$;

3) a does not depend on $f_{2}$;

4) $\bar{f}_{1}, \bar{f}_{3}$ are algebraically independent.

Proof. If $\bar{f}_{2} \in\left\langle\bar{f}_{1}\right\rangle$, then by Lemma 13 we can choose an element $b$ satisfying (13) such that $b \in\left\langle f_{1}\right\rangle$. Then $\left\langle f_{1}, f_{2}\right\rangle=\left\langle f_{1}, g_{2}\right\rangle$. Consequently, there exists a sequence of elementary transformations of the form

$$
\phi=\left(f_{1}, g_{2}, f_{3}\right) \rightarrow\left(f_{1}, g_{2}, f\right) \rightarrow\left(f_{1}, f_{2}, f\right)=\tau .
$$


Since $\operatorname{deg} \phi, \operatorname{deg}\left(f_{1}, g_{2}, f\right)<\operatorname{deg} \theta$ (by (13)), it follows from Corollary 6 that the automorphism $\tau$ is simple.

If $\bar{f}_{3} \in\left\langle\bar{f}_{1}\right\rangle$ and $\bar{f}_{3}=T\left(\bar{f}_{1}\right)$, then we put $g_{3}=f_{3}-T\left(f_{1}\right)$. There exists a sequence

$$
\phi=\left(f_{1}, g_{2}, f_{3}\right) \rightarrow\left(f_{1}, g_{2}, g_{3}\right) \rightarrow\left(f_{1}, f_{2}, g_{3}\right) \rightarrow\left(f_{1}, f_{2}, f_{3}\right)=\theta .
$$

By Corollary 6, the automorphism $\left(f_{1}, f_{2}, g_{3}\right)$ is simple, and consequently, $f_{3}$ is a simple reducible element of $\theta$, which is impossible.

If $a$ does not depend on $f_{2}$, then sequence (14) proves the simplicity of $\tau$.

Assume that $\bar{f}_{1}, \bar{f}_{3}$ are algebraically independent. Then by (13) we obtain that $\bar{f}_{2}=-\bar{b} \in\left\langle\bar{f}_{1}, \bar{f}_{3}\right\rangle$. By 1$)$, we can assume that $\bar{f}_{2} \notin\left\langle\bar{f}_{1}\right\rangle$, i.e., $\bar{f}_{2}$ depends on $\bar{f}_{3}$. Then $\operatorname{deg} f_{3} \leq \operatorname{deg} f_{2}$. Observe that $\bar{f}_{1}, \bar{f}_{2}$ are algebraically independent; otherwise, $\bar{f}_{1}, \bar{f}_{3}$ would be algebraically dependent. Therefore, $\bar{a} \in\left\langle\bar{f}_{1}, \bar{f}_{2}\right\rangle$. By 3 ), we can also assume that $a$ contains $f_{2}$. Then (11) gives $\operatorname{deg} f_{2} \leq \operatorname{deg} a \leq \operatorname{deg} f_{3}$, and so $\operatorname{deg} f_{2}=\operatorname{deg} f_{3}$. Thus

$$
b=\alpha f_{3}+T\left(f_{1}\right), \quad \alpha \neq 0, \quad \operatorname{deg}\left(T\left(f_{1}\right)\right) \leq \operatorname{deg} f_{2} .
$$

We have the equalities

$$
\begin{aligned}
g_{2} & =f_{2}+\alpha f_{3}+T\left(f_{1}\right), \\
f_{2} & =g_{2}-\alpha f_{3}-T\left(f_{1}\right), \\
f_{3} & =\frac{1}{\alpha} g_{2}-\frac{1}{\alpha} f_{2}-\frac{1}{\alpha} T\left(f_{1}\right),
\end{aligned}
$$

which induce the following sequence of elementary transformations:

$$
\left(f_{1}, f_{3}, g_{2}\right) \rightarrow\left(f_{1}, f_{2}, g_{2}\right) \rightarrow\left(f_{1}, f_{2}, f_{3}\right)=\theta .
$$

Since $\phi=\left(f_{1}, g_{2}, f_{3}\right)$ is simple, by Remark 1, $\left(f_{1}, f_{3}, g_{2}\right)$ is simple too. By Lemma 9 . sequence (15) gives simple reducibility of $f_{3}$ in $\theta$, a contradiction.

Thus, by Lemma 14, we can suppose that $\bar{f}_{1}, \bar{f}_{3}$ are algebraically dependent and $\bar{f}_{3} \notin\left\langle\bar{f}_{1}\right\rangle$. We will consider separately the 3 cases:

1) $f_{1}, f_{3}$ is a $*$-reduced pair, $\operatorname{deg} f_{1}<\operatorname{deg} f_{3}$;

2) $f_{1}, f_{3}$ is a $*$-reduced pair, $\operatorname{deg} f_{3}<\operatorname{deg} f_{1}$;

3) $\bar{f}_{1} \in\left\langle\bar{f}_{3}\right\rangle, \operatorname{deg} f_{1}>\operatorname{deg} f_{3}$.

First, we will prove two propositions.

Proposition 4. Let $\psi=\left(g_{1}, g_{2}, g_{3}\right)$ be a simple automorphism satisfying the following conditions:

i) $\operatorname{deg} \psi \leq \operatorname{deg} \theta$;

ii) $g_{1}, g_{2}$ is a 2-reduced pair and $\operatorname{deg} g_{1}=2 n, \operatorname{deg} g_{2}=3 n$;

iii) $\overline{g_{1}}, \overline{g_{3}}$ are linearly independent, $\operatorname{deg} g_{3}=m<3 n$, and $g_{3}$ is not a simple reducible element of $\psi$.

Then one of the following statements is satisfied:

1) $m<n+\operatorname{deg}\left[g_{1}, g_{2}\right]$;

2) $\psi$ admits a reduction of type $I V$ with an active element $g_{3}$; $2 n$.

3) $\operatorname{deg}\left[g_{1}, g_{3}\right]<3 n+\operatorname{deg}\left[g_{1}, g_{2}\right]$, and there exists $\alpha \in F$ such that $\operatorname{deg}\left(g_{2}-\alpha g_{3}^{2}\right) \leq$

Proof. Assume that the proposition is not true, and let $\psi$ be a counterexample of minimal degree. Then we have

$$
n+\operatorname{deg}\left[g_{1}, g_{2}\right] \leq m<3 n .
$$


Hence $\operatorname{deg}\left[g_{1}, g_{2}\right]<2 n$, and by Propositions 1, 2 [3 the automorphism $\psi$ does not admit a reduction of types I-III. By its choice, neither admits $\psi$ a reduction of type IV. So, by the definition of a simple automorphism, $\psi$ admits an elementary reduction. By $i i i)$, either $g_{1}$ or $g_{2}$ is a simple reducible element of $\psi$. Since $\overline{g_{1}}, \overline{g_{2}}$ are algebraically dependent, this implies that $\overline{g_{1}}, \overline{g_{2}}, \overline{g_{3}}$ are mutually algebraically dependent. It follows from iii) and (16) that $g_{1}, g_{3}$ is a $*$-reduced pair.

Case 1. $2 n<m<3 n$.

Assume that $g_{2}$ is a simple reducible element of $\psi$. The inequalities imposed on $m$ make impossible the inclusion $\overline{g_{2}} \in\left\langle\overline{g_{1}}, \overline{g_{3}}\right\rangle$; hence Corollary 1 yields

$$
\operatorname{deg} g_{2}=3 n \geq N\left(g_{1}, g_{3}\right)=\frac{2 n}{(2 n, m)} m-m-2 n+\operatorname{deg}\left[g_{1}, g_{3}\right]
$$

Thus $\frac{2 n}{(2 n, m)} \leq 3$. If $\frac{2 n}{(2 n, m)}=2$, then $m=n t, t \geq 3$ is an odd number, which contradicts (16). Hence $\frac{2 n}{(2 n, m)}=3$. Putting $(2 n, m)=2 \rho$, we have $n=3 \rho$, $\operatorname{deg} g_{1}=6 \rho, m=2 \rho t, t>3,3 \nmid t$. Applying again Corollary 1, we get

$$
9 \rho>6 \rho t-2 \rho t-6 \rho
$$

i.e., $t<\frac{15}{4}$, which is impossible.

If $g_{1}$ is a simple reducible element of $\psi$, then the pair $g_{2}, g_{3}$ is $*$-reduced, and Corollary 1 yields

$$
\operatorname{deg} g_{1}=2 n \geq N\left(g_{2}, g_{3}\right)=\frac{m}{(3 n, m)} 3 n-3 n-m+\operatorname{deg}\left[g_{2}, g_{3}\right] .
$$

Thus $\frac{m}{(3 n, m)}=2$ and $3 n=(3 n, m) t, t \geq 3$ is an odd number. Since $m>2 n$, we have $(3 n, m)>n$ and $3 n=(3 n, m) t \geq(3 n, m) 3>3 n$, a contradiction.

Case 2. $\frac{3 n}{2}<m \leq 2 n$.

Since $\overline{g_{1}}, \overline{g_{3}}$ are algebraically dependent, they would be linearly dependent if $m=2 n$, which contradicts $i$ ii). Therefore, $m<2 n$. If $g_{2}$ is a simple reducible element of $\psi$, then Corollary 1 gives

$$
\operatorname{deg} g_{2}=3 n \geq N\left(g_{3}, g_{1}\right)=\frac{m}{(2 n, m)} 2 n-2 n-m+\operatorname{deg}\left[g_{1}, g_{3}\right] .
$$

Therefore, $\frac{m}{(2 n, m)} \leq 3$. If $\frac{m}{(2 n, m)}=2$, then $2 n=(2 n, m) t, t \geq 3$ is an odd number. Hence $m=\frac{4 n}{t}$, and the inequality $m>\frac{3 n}{2}$ yields $t<\frac{8}{3}$, which is impossible. If $\frac{m}{(2 n, m)}=3$, then $2 n=(2 n, m) t, t>3,3 \not t$. Thus $m=\frac{6 n}{t}>\frac{3 n}{2}$ and $t<4$, which is also impossible.

If $g_{1}$ is a simple reducible element of $\psi$, then it follows from (17) that $\frac{m}{(3 n, m)}=2$, $3 n=(3 n, m) t, t \geq 3$ is an odd number. Since $\frac{3 n}{2}<m=\frac{6 n}{t}<2 n$, then $3<t<4$, which is impossible. So case 2 is done.

Now we can assume that $m \leq \frac{3 n}{2}$. Then (16) gives $\operatorname{deg}\left[g_{1}, g_{2}\right] \leq \frac{n}{2}$, and consequently,

$$
\operatorname{deg}\left[g_{1}, g_{2}\right]+\operatorname{deg} g_{3} \leq 2 n .
$$

Then, by Lemma 1, we have

$$
\operatorname{deg}\left[g_{2}, g_{3}\right]+\operatorname{deg} g_{1}=\operatorname{deg}\left[g_{1}, g_{3}\right]+\operatorname{deg} g_{2},
$$

i.e.,

$$
\operatorname{deg}\left[g_{2}, g_{3}\right]=\operatorname{deg}\left[g_{1}, g_{3}\right]+n
$$

Case 3. $m=\frac{3 n}{2}$. 
By putting $n=2 \rho$, we have $\operatorname{deg} g_{1}=4 \rho, \operatorname{deg} g_{2}=6 \rho, \operatorname{deg} g_{3}=3 \rho$. Put also $\overline{g_{2}}=\alpha{\overline{g_{3}}}^{2}$ (recall that $\bar{g}_{2}$ and $\bar{g}_{3}$ are algebraically dependent). We will first show that $g_{2}$ is a simple reducible element of $\psi$. Assume that $g_{1}$ is a simple reducible element of $\psi$ which is reduced by a simple automorphism $\psi_{1}=\left(h_{1}, g_{2}, g_{3}\right)$. Then $\operatorname{deg} \psi_{1}<\operatorname{deg} \theta$, and by Lemma 9 the automorphism $\psi_{2}=\left(h_{1}, g_{2}-\alpha g_{3}^{2}, g_{3}\right)$ is also simple. The sequence

$$
\psi_{2} \rightarrow\left(g_{1}, g_{2}-\alpha g_{3}^{2}, g_{3}\right) \rightarrow\left(g_{1}, g_{2}, g_{3}\right)=\psi
$$

proves that $g_{2}$ is a simple reducible element of $\psi$.

Put

$$
h_{2}=g_{2}-T\left(g_{3}, g_{1}\right), \operatorname{deg} h_{2}<\operatorname{deg} g_{2},
$$

where $h_{2}$ is an unreducible element of $\xi=\left(g_{1}, h_{2}, g_{3}\right)$. Let $\operatorname{deg}_{y}(T(x, y))=k=$ $3 q+r, 0 \leq r<3$. Since $\operatorname{deg}\left(T\left(g_{3}, g_{1}\right)\right)=\operatorname{deg} g_{2}=6 \rho$, inequality (2) yields that either $q=1, r=0$, or $q=0, r=0,1$.

Consider first the case $q=1$. Note that $g_{3}, g_{1}$ is a 3 -reduced pair and $\operatorname{deg}_{y}(w(x, y))=3$, where $w(x, y)$ is a derivative polynomial of the pair $g_{3}, g_{1}$. By Lemma 7, the polynomial $T(x, y)$ can be presented in the form

$$
T(x, y)=w(x, y) q(x, y)+s(x, y)
$$

where $\operatorname{deg}_{y}(q(x, y))=0, \operatorname{deg}_{y}(s(x, y))<3$. By Lemma 6.3),

$$
\operatorname{deg}\left(w\left(g_{3}, g_{1}\right)\right) \geq N\left(g_{3}, g_{1}\right)=3 \cdot 4 \rho-4 \rho-3 \rho+\operatorname{deg}\left[g_{1}, g_{3}\right]>5 \rho .
$$

Since $\left.\operatorname{deg}\left(T\left(g_{3}, g_{1}\right)\right)=6 \rho, \operatorname{Lemma} 7.2\right)$ gives $\operatorname{deg}\left(q\left(g_{3}, g_{1}\right)\right)<\rho, \operatorname{deg}\left(s\left(g_{3}, g_{1}\right)\right) \leq 6 \rho$. The polynomials $q(x, y), s(x, y)$ satisfy condition (ii) of Corollary 1. Hence they satisfy also (iii), and we get $q(x, y)=\lambda \neq 0, s(x, y)=\gamma x^{2}+\delta x+\mu y$. Therefore,

$$
T(x, y)=\lambda w(x, y)+\gamma x^{2}+\delta x+\mu y \text {. }
$$

Consequently, by Corollary 3 ,

$$
\operatorname{deg}\left(\frac{\partial T}{\partial x}\left(g_{3}, g_{1}\right)\right)=9 \rho, \operatorname{deg}\left(\frac{\partial T}{\partial y}\left(g_{3}, g_{1}\right)\right)=8 \rho .
$$

By (1) and (20),

$$
\begin{aligned}
& {\left[g_{1}, h_{2}\right]=\left[g_{1}, g_{2}\right]-\left[g_{1}, g_{3}\right] \frac{\partial T}{\partial x}\left(g_{3}, g_{1}\right),} \\
& {\left[h_{2}, g_{3}\right]=\left[g_{2}, g_{3}\right]-\left[g_{1}, g_{3}\right] \frac{\partial T}{\partial y}\left(g_{3}, g_{1}\right) .}
\end{aligned}
$$

This yields, by (16) and (19),

$$
\begin{aligned}
\operatorname{deg}\left[g_{1}, h_{2}\right] & =\operatorname{deg}\left[g_{1}, g_{3}\right]+9 \rho, \\
\operatorname{deg}\left[h_{2}, g_{3}\right] & =\operatorname{deg}\left[g_{1}, g_{3}\right]+8 \rho .
\end{aligned}
$$

Therefore, $\operatorname{deg} h_{2} \geq \operatorname{deg}\left[g_{1}, g_{3}\right]+5 \rho$. Since $\operatorname{deg} h_{2}<\operatorname{deg} g_{2}=6 \rho$, this yields $\operatorname{deg}\left[g_{1}, g_{3}\right]<\rho$. Now, applying F2) and Corollary 1, it is easy to show that $g_{1}, g_{3}$ are unreducible elements of $\xi=\left(g_{1}, h_{2}, g_{3}\right)$. Since $\operatorname{deg}\left[g_{1}, g_{3}\right]<\rho$, by Propositions 1 . 213 we conclude that $\xi$ does not admit reductions of types I-IV. This contradicts the simplicity of $\xi$.

Hence $q=0, r=0,1$. By Corollary 1 ( (ii) implies (iii) ), we have

$$
T(x, y)=\alpha x^{2}+\beta x+\gamma y \text {. }
$$


Then $\operatorname{deg}\left(\frac{\partial T}{\partial y}\left(g_{3}, g_{1}\right)\right) \leq 0$ and by (19), (22), we conclude that

$$
\operatorname{deg}\left[h_{2}, g_{3}\right]=\operatorname{deg}\left[g_{2}, g_{3}\right]=\operatorname{deg}\left[g_{1}, g_{3}\right]+2 \rho .
$$

Now we will assume that $\operatorname{deg} h_{2}>2 n$ and show that this case is impossible. If $g_{3}$ is a simple reducible element of $\xi$, then $g_{1}, h_{2}$ form a $*$-reduced pair, and it follows easily from (2) that this pair is 2-reduced. But it is impossible since $\operatorname{deg} h_{2}<3 n$. Furthermore, if $g_{1}$ is a simple reducible element of $\xi$, then $g_{3}, h_{2}$ form a 2-reduced pair, that is, $\operatorname{deg} h_{2}=\frac{9}{2} \rho$. Put

$$
h_{1}=g_{1}+Q\left(g_{3}, h_{2}\right), \operatorname{deg} h_{1}<\operatorname{deg} g_{1},
$$

where $h_{1}$ is an unreducible element of the simple automorphism $\xi_{1}=\left(h_{1}, h_{2}, g_{3}\right)$.

Observe that $\xi_{1}$ satisfies all the conditions of the present proposition. By (2) and (23), we get $\operatorname{deg}_{y}(Q(x, y))=2$. Lemma 74 ) and Corollary 3 give

$$
\operatorname{deg}\left(\frac{\partial Q}{\partial y}\left(g_{3}, h_{2}\right)\right)=\operatorname{deg} h_{2}=\frac{9}{2} \rho .
$$

Since

$$
\left[h_{1}, g_{3}\right]=\left[g_{1}, g_{3}\right]+\left[h_{2}, g_{3}\right] \frac{\partial Q}{\partial y}\left(g_{3}, h_{2}\right)
$$

by (23) we have

$$
\operatorname{deg}\left[h_{1}, g_{3}\right]=\operatorname{deg}\left[h_{2}, g_{3}\right]+\frac{9}{2} \rho .
$$

Therefore,

$$
\operatorname{deg} h_{1} \geq \operatorname{deg}\left[h_{2}, g_{3}\right]+\frac{3}{2} \rho=\frac{7}{2} \rho+\operatorname{deg}\left[g_{1}, g_{3}\right] .
$$

These inequalities show that $\xi_{1}$ does not satisfy the conclusions of the proposition. Since $\operatorname{deg} \xi_{1}<\operatorname{deg} \psi$, this contradicts our choice of $\psi$.

We have thus shown that $\xi$ does not admit elementary reductions. As for reductions of type I, the only possibility for $\xi$ to have it is when $g_{1}$ is an active element of reduction and $\operatorname{deg} h_{2}=\frac{9}{2} \rho$. Then there should exist $\alpha_{1} \in F$ such that the element $g_{1}$ of the automorphism $\left(g_{1}, h_{2}-\alpha_{1} g_{1}, g_{3}\right)$ is reducible. Recall that $\underline{h_{2}}=g_{2}-\alpha g_{3}^{2}-\beta g_{3}-\gamma g_{1}$. Set $h_{2}^{\prime}=h_{2}-\alpha_{1} g_{1}=g_{2}-\alpha g_{3}^{2}-\beta g_{3}-\left(\gamma+\alpha_{1}\right) g_{1}$. Then $\overline{h_{2}^{\prime}}=\overline{h_{2}}$ and we can replace $h_{2}$ by $h_{2}^{\prime}$ in our previous arguments. Then, as above, the condition for $g_{1}$ to be a reducible element of $\left(g_{1}, h_{2}^{\prime}, g_{3}\right)$ gives a contradiction.

Furthermore, the comparison of the degrees of the components of $\xi$ shows that $\xi$ does not admit a reduction of type II. Thus $\xi$ admits a reduction of type III or IV, with an active element $g_{3}$. Let $\left(r_{1}, r_{2}, r_{3}\right)$ be a reduction of type III or a predreduction of type IV of $\xi$,

$$
r_{1}=g_{1}-\beta_{1} g_{3}, \quad r_{2}=h_{2}-\alpha_{1} g_{3}^{2}-\gamma_{1} g_{3} .
$$

Observe that, by definition, $\operatorname{deg} r_{1}=4 \rho, \operatorname{deg} r_{2}=6 \rho$. We have

$$
\begin{aligned}
r_{2}= & g_{2}-\left(\alpha+\alpha_{1}\right) g_{3}^{2}-\left(\beta+\gamma_{1}\right) g_{3}-\gamma g_{1}, \\
{\left[r_{1}, r_{2}\right]=} & {\left[g_{1}, g_{2}\right]-2\left(\alpha+\alpha_{1}\right)\left[g_{1}, g_{3}\right] g_{3} } \\
& -\left(\beta+\gamma_{1}+\gamma \beta_{1}\right)\left[g_{1}, g_{3}\right]+\beta_{1}\left[g_{2}, g_{3}\right] .
\end{aligned}
$$


By Proposition[3, applied to the triple $\xi=\left(g_{1}, h_{2}, g_{3}\right), \operatorname{deg}\left[r_{1}, r_{2}\right] \leq \rho, \operatorname{deg}\left[g_{1}, g_{3}\right]>$ $6 \rho$. Furthermore, by (16) and (19), $\operatorname{deg}\left[g_{1}, g_{2}\right] \leq \rho, \operatorname{deg}\left[g_{1}, g_{3}\right]>8 \rho$. Comparing the degrees of the left and right parts in the last equation gives

$$
\alpha+\alpha_{1}=0, \quad \beta+\gamma_{1}+\gamma \beta_{1}=0, \quad \beta_{1}=0 .
$$

Thus $r_{1}=g_{1}, r_{2}=g_{2}-\gamma g_{1}, r_{3}=\sigma g_{3}+g$, where $g \in\left\langle r_{1}, r_{2}\right\rangle \backslash F$. Since $g_{3}$ is unreducible in $\psi, \operatorname{deg} r_{3}=\operatorname{deg} g_{3}=3 \rho$. But then

$$
\operatorname{deg} r_{1}+\operatorname{deg} r_{2}+\operatorname{deg} r_{3}=13 \rho>\operatorname{deg} g_{1}+\operatorname{deg} h_{2}+\operatorname{deg} g_{3}=\operatorname{deg} \xi,
$$

which contradicts Corollary 4 .

Therefore, the automorphism $\left(r_{1}, r_{2}, r_{3}\right)$ is not a reduction of type III of $\xi$. Assume that it is a predreduction of type IV of $\xi$; then it is easy to see that in this case $\psi$ also admits a reduction of type IV with an active element $g_{3}$, which is impossible.

We have thus shown that the inequality $\operatorname{deg} h_{2}>2 n$ is impossible. Hence $\operatorname{deg} h_{2} \leq 2 n$. Since $h_{2}=g_{2}-\alpha g_{3}^{2}-\beta g_{3}-\gamma g_{1}$, this implies $\operatorname{deg}\left(g_{2}-\alpha g_{3}^{2}\right) \leq 2 n$. Furthermore, it follows from (23) that

$$
\operatorname{deg}\left[g_{1}, g_{3}\right]+2 \rho \leq \operatorname{deg} h_{2}+\operatorname{deg} g_{3} \leq 7 \rho,
$$

i.e., $\operatorname{deg}\left[g_{1}, g_{3}\right] \leq 5 \rho$. Thus the automorphism $\psi$ satisfies statement 3$)$ of the present proposition, which is impossible.

Case 4. $m<\frac{3 n}{2}$.

If $g_{1}$ is a simple reducible element of $\psi$, then (17) gives that $\frac{m}{(3 n, m)}=2$. Applying once more (17) and (19), we get

$$
2 n \geq 3 n-m+\operatorname{deg}\left[g_{2}, g_{3}\right]=4 n-m+\operatorname{deg}\left[g_{1}, g_{3}\right],
$$

i.e., $m>2 n$, a contradiction.

Therefore, $g_{2}$ is a simple reducible element of $\psi$. By (18), $\frac{m}{(2 n, m)} \leq 3$.

Consider first the case $\frac{m}{(2 n, m)}=3$. Then $2 n=(2 n, m) t, t>3,3 \nmid t$. Hence $m=\frac{6 n}{t}$, and since $n<m<\frac{3 n}{2}$, we have $t=5$. By putting $n=5 \rho$, we have $\operatorname{deg} g_{1}=10 \rho, \operatorname{deg} g_{2}=15 \rho, \operatorname{deg} g_{3}=6 \rho$. Then (18) gives $\operatorname{deg}\left[g_{1}, g_{3}\right] \leq \rho$.

Consider equality (20) and put again $\operatorname{deg}_{y} T(x, y)=k=3 q+r, 0 \leq r<3$. Since $\operatorname{deg}\left(T\left(g_{3}, g_{1}\right)\right)=\operatorname{deg} g_{2}=15 \rho$, inequality (2) yields that either $q=0, r=1$ or $q=1, r=0$. In the first case, by Corollary $1, \overline{T\left(g_{3}, g_{1}\right)}=\bar{g}_{2} \in\left\langle\bar{g}_{1}, \bar{g}_{3}\right\rangle$, which is impossible. Thus $k=3$ and by Lemma [74) $T(x, y)$ is a derivative polynomial (up to a nonzero scalar multiplier) of the pair $g_{3}, g_{1}$. Then Corollary 3 gives

$$
\operatorname{deg}\left(\frac{\partial T}{\partial x}\left(g_{3}, g_{1}\right)\right)=24 \rho, \operatorname{deg}\left(\frac{\partial T}{\partial y}\left(g_{3}, g_{1}\right)\right)=20 \rho .
$$

From (21), (22), taking into account (16) and (19), we get

$$
\begin{aligned}
\operatorname{deg}\left[g_{1}, h_{2}\right] & =\operatorname{deg}\left[g_{1}, g_{3}\right]+24 \rho, \\
\operatorname{deg}\left[h_{2}, g_{3}\right] & =\operatorname{deg}\left[g_{1}, g_{3}\right]+20 \rho .
\end{aligned}
$$

Hence $15 \rho>\operatorname{deg} h_{2} \geq 14 \rho+\operatorname{deg}\left[g_{1}, g_{3}\right]$, and Corollary 1 yields that $g_{1}, g_{3}$ are unreducible elements of the automorphism $\xi=\left(g_{1}, h_{2}, g_{3}\right)$. Since $\operatorname{deg}\left[g_{1}, g_{3}\right]<\rho$, it is easy to check that $\xi$ does not admit reductions of types I-IV, which contradicts the simplicity of $\xi$.

Now we consider the case $\frac{m}{(2 n, m)}=2$. By putting $(2 n, m)=2 \rho$, we get $m=4 \rho$, $2 n=2 \rho t, t \geq 3$ is an odd number. Then $m=\frac{4 n}{t}$ and (16) implies $t<4$. Hence $t=3$ and $\operatorname{deg} g_{1}=6 \rho, \operatorname{deg} g_{2}=9 \rho, \operatorname{deg} g_{3}=4 \rho$. Consider again equality (20) and 
put $\operatorname{deg}_{y}(T(x, y))=k=2 q+r, 0 \leq r<2$. Assume that $r=1$. Then (2) yields $q=1$, i.e., $k=3$. By Lemma 7

$$
T(x, y)=w(x, y) q(x, y)+s(x, y),
$$

where $w(x, y)$ is a derivative polynomial of the 2-reduced pair $g_{3}, g_{1}$, and $\operatorname{deg}_{y}(q(x, y))=1, \operatorname{deg}_{y}(s(x, y)) \leq 1$. By Lemma [5)

$$
\operatorname{deg}\left(w\left(g_{3}, g_{1}\right)\right) \geq N\left(g_{3}, g_{1}\right)=12 \rho-6 \rho-4 \rho+\operatorname{deg}\left[g_{1}, g_{3}\right]>2 \rho .
$$

Now Lemma 7,2) gives $\operatorname{deg}\left(q\left(g_{3}, g_{1}\right)\right)<7 \rho$, and then Lemma 3 yields $\operatorname{deg}\left(q\left(g_{3}, g_{1}\right)\right)$ $=6 \rho$. Thus, by Lemma 73$)$, we get

$$
\operatorname{deg}\left(\frac{\partial T}{\partial x}\left(g_{3}, g_{1}\right)\right)=14 \rho, \operatorname{deg}\left(\frac{\partial T}{\partial y}\left(g_{3}, g_{1}\right)\right)=12 \rho .
$$

Now (21) and (22) yield, by means of (16) and (19),

$$
\begin{aligned}
\operatorname{deg}\left[g_{1}, h_{2}\right] & =\operatorname{deg}\left[g_{1}, g_{3}\right]+14 \rho, \\
\operatorname{deg}\left[h_{2}, g_{3}\right] & =\operatorname{deg}\left[g_{1}, g_{3}\right]+12 \rho .
\end{aligned}
$$

Hence $\operatorname{deg} h_{2} \geq 8 \rho+\operatorname{deg}\left[g_{1}, g_{3}\right]$, and the elements $g_{1}, g_{3}$ of $\xi=\left(g_{1}, h_{2}, g_{3}\right)$ are unreducible. Since $\operatorname{deg}\left[g_{1}, g_{3}\right]<\rho$, it is easy to check that $\xi$ does not admit reductions of type I-IV, a contradiction.

Thus $r=0, k=2 q$. Inequality (2) gives $1 \leq q \leq 4$ and

$$
\operatorname{deg}\left(\frac{\partial T}{\partial y}\left(g_{3}, g_{1}\right)\right) \geq(q-1)\left(2 \rho+\operatorname{deg}\left[g_{3}, g_{1}\right]\right)+6 \rho .
$$

Hence, by means of (19), (22),

$$
\operatorname{deg}\left[h_{2}, g_{3}\right] \geq q\left(2 \rho+\operatorname{deg}\left[g_{3}, g_{1}\right]\right)+4 \rho .
$$

In particular,

$$
\operatorname{deg} h_{2} \geq q\left(2 \rho+\operatorname{deg}\left[g_{3}, g_{1}\right]\right) .
$$

Assume that $\operatorname{deg} h_{2} \geq 6 \rho$. Since $h_{2}$ is an unreducible element of $\xi=\left(g_{1}, h_{2}, g_{3}\right)$, the elements $\overline{g_{1}}, \bar{h}_{2}$ are linearly independent, if $\operatorname{deg} h_{2}=6 \rho$. Note that $g_{1}, h_{2}$ and $g_{3}, h_{2}$ do not compose 2-reduced pairs. Consequently, the elements $g_{1}, g_{3}$ of $\xi$ are unreducible. In fact, assume that there exists $f \in\left\langle h_{2}, g_{3}\right\rangle$ such that $\bar{g}_{1}=\bar{f}$. Since $\bar{g}_{1} \notin\left\langle\bar{h}_{2}, \bar{g}_{3}\right\rangle$, the elements $\bar{h}_{2}, \bar{g}_{3}$ are algebraically dependent and the pair $g_{3}, h_{2}$ is *-reduced. It follows easily from (2) that this pair should be 2-reduced, a contradiction. Similarly, $g_{3}$ is unreducible. Furthermore, it follows from (25) that $\operatorname{deg}\left[g_{3}, g_{1}\right]<\operatorname{deg} h_{2}$. Hence, due to Definitions 114 and Propositions 10 3, $\xi$ does not admit reductions of types I-IV. This contradicts the simplicity of $\xi$.

Therefore, $\operatorname{deg} h_{2}<6 \rho$ and $\xi$ satisfies all the conditions of the present proposition. Since $\operatorname{deg} \xi<\operatorname{deg} \psi$, then, by the choice of $\psi, \xi$ should satisfy the conclusion of the proposition. It follows from (24), (25) that $\xi$ does not satisfy statements $1), 3$ ); hence $\xi$ admits a reduction of type IV with an active element $h_{2}$. In this case, $\operatorname{deg} h_{2} \leq 3 \rho$ and we have $q=1$ in (25), which implies $\operatorname{deg}\left[g_{1}, g_{3}\right] \leq \rho$. By statement 5) of Proposition 3, $\xi$ has a predreduction of the form $\left(g_{1}, \tilde{h}_{2}, g_{3}\right)$, where

$$
\tilde{h}_{2}=\sigma h_{2}+g, g \in\left\langle g_{3}, g_{1}\right\rangle \backslash F, \operatorname{deg} \tilde{h}_{2}=3 \rho, \operatorname{deg}\left[g_{3}, \tilde{h}_{2}\right]<6 \rho+\operatorname{deg}\left[g_{3}, g_{1}\right] \text {. }
$$

By Corollary 2 we have $g=\lambda w\left(g_{3}, g_{1}\right)$, where $w(x, y)$ is a derivative polynomial of the pair $g_{3}, g_{1}$. Then $\operatorname{deg}_{y}(w(x, y))=2$. Now $\tilde{h}_{2}=\sigma\left(g_{2}-T\left(g_{3}, g_{1}\right)\right)+\lambda w\left(g_{3}, g_{1}\right)$ and $\sigma^{-1} \tilde{h}_{2}=g_{2}-\tilde{T}\left(g_{3}, g_{1}\right)$, where $\tilde{T}(x, y)=T(x, y)-\sigma^{-1} \lambda w(x, y)$. If we substitute 
$\sigma^{-1} \tilde{h}_{2}$ instead of $h_{2}$ in (20) (with $\tilde{T}$ instead of $T$ ), we will have again $\operatorname{deg}_{y}(\tilde{T}(x, y))=$ $2 q, 1 \leq q \leq 4$, since the equality $\operatorname{deg}_{y}(\tilde{T}(x, y))=3$ is impossible. Then inequalities (24) and (25) hold also for $\tilde{h}_{2}$. In particular,

$$
\operatorname{deg}\left[g_{3}, \tilde{h}_{2}\right] \geq 6 \rho+\operatorname{deg}\left[g_{3}, g_{1}\right],
$$

which contradicts the previous inequality.

Proposition 5. Let $\psi=\left(g_{1}, g_{2}, g_{3}\right)$ be a simple automorphism satisfying the following conditions:

i) $\operatorname{deg} \psi \leq \operatorname{deg} \theta$;

ii) $g_{1}, g_{2}$ is a 2-reduced pair and $\operatorname{deg} g_{1}=2 n$, $\operatorname{deg} g_{2}=n s$, where $s \geq 5$ is an odd number;

iii) $\overline{g_{3}} \notin\left\langle\overline{g_{1}}\right\rangle, \operatorname{deg} g_{3}=m<n s$, and $g_{3}$ is not a simple reducible element of $\psi$.

Then $m<n(s-2)+\operatorname{deg}\left[g_{1}, g_{2}\right]$.

Proof. Assuming the contrary, we have

$$
n(s-2)+\operatorname{deg}\left[g_{1}, g_{2}\right] \leq m<n s .
$$

Hence $\operatorname{deg}\left[g_{1}, g_{2}\right]<2 n$. It is now easy to check that $\psi$ does not admit reductions of type I-IV. Then $\psi$ is elementary reducible, and either $g_{1}$ or $g_{2}$ is a simple reducible element of $\psi$. In particular, $\overline{g_{1}}, \overline{g_{2}}, \overline{g_{3}}$ are mutually algebraically dependent. It follows from (26) that $\overline{g_{2}} \notin\left\langle\overline{g_{1}}, \overline{g_{3}}\right\rangle, \overline{g_{1}} \notin\left\langle\overline{g_{2}}, \overline{g_{3}}\right\rangle$. Consequently, $g_{1}, g_{3}$ and $g_{2}, g_{3}$ are $*$-reduced pairs as well.

If $g_{2}$ is a simple reducible element of $\psi$, then by Corollary $\square$ we get

$$
\operatorname{deg} g_{2}=n s \geq N\left(g_{1}, g_{3}\right)=\frac{2 n}{(2 n, m)} m-m-2 n+\operatorname{deg}\left[g_{1}, g_{3}\right] .
$$

Observe that by Lemma 2.i) we have $p=\frac{2 n}{(2 n, m)} \geq 2$. If $\frac{2 n}{(2 n, m)} \geq 4$, inequalities (26), (27) give $n s>3 m-2 n>3 n(s-2)-2 n$. Hence $s<4$, which contradicts condition $i$ ) of the proposition.

If $\frac{2 n}{(2 n, m)}=2$, then $n=(2 n, m), m=n t$, where $t \geq 3$ is an odd number. From (26) we get $s-2<t<s$, which is impossible.

Therefore, $\frac{2 n}{(2 n, m)}=3$. By putting $(2 n, m)=2 \rho$, we have $n=3 \rho, \operatorname{deg} g_{1}=6 \rho$, $m=2 \rho t$, where $t>3,3 \chi t$. Inequalities (26) and (27) yield

$$
3(s+2)>4 t, \quad 3(s-2)<2 t<3 s .
$$

Hence $t=s=5$, i.e., $\operatorname{deg} g_{1}=6 \rho, \operatorname{deg} g_{2}=15 \rho, \operatorname{deg} g_{3}=10 \rho$. Applying (26), (27) once more, we get

$$
\operatorname{deg}\left[g_{1}, g_{2}\right] \leq \rho, \operatorname{deg}\left[g_{1}, g_{3}\right] \leq \rho .
$$

Let again $h_{2}=g_{2}-T\left(g_{1}, g_{3}\right)$, where $\operatorname{deg} h_{2}<\operatorname{deg} g_{2}$ and $h_{2}$ is unreducible in $\xi=\left(g_{1}, h_{2}, g_{3}\right)$. Since $\operatorname{deg}\left(T\left(g_{1}, g_{3}\right)\right)=\operatorname{deg} g_{2}=15 \rho$ and $\overline{T\left(g_{1}, g_{3}\right)}=\bar{g}_{2} \notin\left\langle\bar{g}_{1}, \bar{g}_{3}\right\rangle$, inequality (2) yields $\operatorname{deg}_{y}(T(x, y))=3$. By Lemma 7.4), up to a scalar multiplier, $T(x, y)$ is equal to a derivative polynomial of the pair $g_{1}, g_{3}$. Hence by Corollary 3

$$
\operatorname{deg}\left(\frac{\partial T}{\partial x}\left(g_{1}, g_{3}\right)\right)=24 \rho, \operatorname{deg}\left(\frac{\partial T}{\partial y}\left(g_{1}, g_{3}\right)\right)=20 \rho .
$$

Since

$$
\begin{aligned}
\operatorname{deg}\left[g_{1}, g_{3}\right]+\operatorname{deg} g_{2} & \leq 16 \rho, \\
\operatorname{deg}\left[g_{1}, g_{2}\right]+\operatorname{deg} g_{3} & \leq 11 \rho,
\end{aligned}
$$


Lemma 1 implies that

$$
\operatorname{deg}\left[g_{2}, g_{3}\right]+\operatorname{deg} g_{1} \leq 16 \rho .
$$

Therefore, $\operatorname{deg}\left[g_{2}, g_{3}\right] \leq 10 \rho$. Now equalities (21), (22), with $g_{1}$ and $g_{3}$ permuted, give

$$
\begin{aligned}
\operatorname{deg}\left[g_{1}, h_{2}\right] & =\operatorname{deg}\left[g_{1}, g_{3}\right]+20 \rho, \\
\operatorname{deg}\left[h_{2}, g_{3}\right] & =\operatorname{deg}\left[g_{1}, g_{3}\right]+24 \rho .
\end{aligned}
$$

Hence $\operatorname{deg} h_{2} \geq 14 \rho+\operatorname{deg}\left[g_{1}, g_{3}\right]$, which implies easily by (2) that the elements $g_{1}, g_{3}$ are unreducible in $\xi=\left(g_{1}, h_{2}, g_{3}\right)$. Since $\operatorname{deg}\left[g_{1}, g_{3}\right] \leq \rho$, it is easily checked that $\xi$ does not admit reductions of types I-IV. Thus, $\xi$ is not a simple automorphism.

Now suppose that $g_{1}$ is a simple reducible element of $\psi$. Then Corollary 1 gives

$$
\operatorname{deg} g_{1}=2 n \geq N\left(g_{3}, g_{2}\right)=\frac{m}{(n s, m)} n s-n s-m+\operatorname{deg}\left[g_{2}, g_{3}\right] .
$$

Therefore, $\frac{m}{(n s, m)}=2$ and $n s=(n s, m) t$, where $t \geq 3$ is an odd number. Then $m=\frac{2 n s}{t}$ and (26) gives $s-2<\frac{2 s}{t}$, i.e., $(t-2)(s-2)<4$. Since $s \geq 5$, this yields $t=3, s=5$. By putting $(n s, m)=5 \rho$, we have again $\operatorname{deg} g_{1}=6 \rho, \operatorname{deg} g_{2}=15 \rho$, $\operatorname{deg} g_{3}=10 \rho$. By (26), we have also $\operatorname{deg}\left[g_{1}, g_{2}\right] \leq \rho$. Suppose that

$$
h_{1}=g_{1}+G\left(g_{3}, g_{2}\right), \quad \operatorname{deg} h_{1}<\operatorname{deg} g_{1},
$$

where $h_{1}$ is an unreducible element of $\xi=\left(h_{1}, g_{2}, g_{3}\right)$. Since $\operatorname{deg}\left(G\left(g_{3}, g_{2}\right)\right)=$ $\operatorname{deg} g_{1}=6 \rho$, by Corollary 2 we have $G(x, y)=\lambda w(x, y)$, where $w(x, y)$ is a derivative polynomial of the pair $g_{3}, g_{2}$. Therefore, by Corollary 3 .

$$
\operatorname{deg}\left(\frac{\partial G}{\partial x}\left(g_{3}, g_{2}\right)\right)=2 \cdot \operatorname{deg} g_{3}=20 \rho .
$$

Since

$$
\left[h_{1}, g_{2}\right]=\left[g_{1}, g_{2}\right]+\left[g_{3}, g_{2}\right] \frac{\partial G}{\partial x}\left(g_{3}, g_{2}\right),
$$

a comparison of degrees gives

$$
\operatorname{deg}\left[h_{1}, g_{2}\right]=\operatorname{deg}\left[g_{3}, g_{2}\right]+20 \rho .
$$

Thus,

$$
\operatorname{deg} h_{1} \geq 5 \rho+\operatorname{deg}\left[g_{3}, g_{2}\right], \quad \operatorname{deg}\left[g_{3}, g_{2}\right]<\rho .
$$

It remains to note that $\xi=\left(h_{1}, g_{2}, g_{3}\right)$ satisfies all the conditions of Proposition 4 and does not satisfy conclusions 1), 3) of this proposition. If $\xi$ had admitted a reduction of type IV with an active element $h_{1}$, then by the definition there would exist an element $g \in\left\langle g_{3}, g_{2}\right\rangle \backslash F$ such that $\operatorname{deg}\left(h_{1}+g\right)=\frac{15 \rho}{2}$, i.e., $\operatorname{deg} g=\frac{15 \rho}{2}$. Then by Corollary 2 we would have again $g=\alpha_{1} w\left(g_{3}, g_{2}\right)$, which contradicts the equality $\operatorname{deg}\left(w\left(g_{3}, g_{2}\right)\right)=\operatorname{deg}\left(G\left(g_{3}, g_{2}\right)\right)=6 \rho$.

Lemma 15. If $f_{1}, f_{3}$ is a $*$-reduced pair and $\operatorname{deg} f_{1}<\operatorname{deg} f_{3}$, then $\tau$ is a simple automorphism.

Proof. We consider first the case when $\operatorname{deg} f_{2}>\operatorname{deg} f_{3}$. By (11), we have $a \in$ $\left\langle f_{1}, f_{2}\right\rangle, \operatorname{deg} a \leq \operatorname{deg} f_{3}$. By Lemma 14, we may assume that $\bar{f}_{2} \notin\left\langle\bar{f}_{1}\right\rangle$ and $a \notin\left\langle f_{1}\right\rangle$. Since $\operatorname{deg} a<\operatorname{deg} f_{2}$, then by F2), $\bar{f}_{1}, \bar{f}_{2}$ are algebraically dependent. By Corollary 
1] $\operatorname{deg} a \geq N=N\left(f_{1}, f_{2}\right)$. Hence $\operatorname{deg} f_{2}>N$ and by Lemma 2 2 iii), the pair $f_{1}, f_{2}$ is 2-reduced, that is, $\operatorname{deg} f_{1}=2 n, \operatorname{deg} f_{2}=n s$, where $s \geq 3$ is an odd number, and

$$
n(s-2)+\operatorname{deg}\left[f_{1}, f_{2}\right] \leq \operatorname{deg} a \leq \operatorname{deg} f_{3} .
$$

Since $f_{3}$ is not a simple reducible element of $\theta$ (see Corollary 17), $\theta$ satisfies the conditions of one of Propositions 4 5. Since $\operatorname{deg} f_{3}>\operatorname{deg} f_{1}$, statements 2), 3) of Proposition 4 are not fulfilled for $\theta$. Therefore,

$$
\operatorname{deg} f_{3}<n(s-2)+\operatorname{deg}\left[f_{1}, f_{2}\right],
$$

which contradicts the previous inequality.

Suppose now that $\operatorname{deg} f_{2} \leq \operatorname{deg} f_{3}$. If $\operatorname{deg} f_{2}=\operatorname{deg} f_{3}$ and $\bar{f}_{2}, \bar{f}_{3}$ are linearly dependent, then the element $b$ in (13) can be chosen as $b=\alpha f_{3}$. Then sequence (15) gives the simplicity of $\tau$.

Thus we can assume that $\operatorname{deg} f_{2}<\operatorname{deg} f_{3}$. Then using Lemma 14i) we can furthermore assume that $\bar{b}=-\bar{f}_{2} \notin\left\langle\bar{f}_{1}, \bar{f}_{3}\right\rangle$. Since $\operatorname{deg} b \leq \operatorname{deg} f_{3}$, by Corollary 1 $N\left(f_{1}, f_{3}\right) \leq \operatorname{deg} f_{3}$, and by Lemma 2 iii) the elements $f_{1}, f_{3}$ form a 2 -reduced pair. Put $\operatorname{deg} f_{1}=2 n, \operatorname{deg} f_{3}=n s$, where $s \geq 3$ is an odd number. The inequality $\operatorname{deg} f_{3}=n s \geq N\left(f_{1}, f_{3}\right)$ gives also $\operatorname{deg}\left[f_{1}, f_{3}\right] \leq 2 n$. We can also assume that $g_{2}$ is an unreducible element of $\phi=\left(f_{1}, g_{2}, f_{3}\right)$. Then $\phi$ satisfies the conditions of one of Propositions 4, 5,

Let $b=T\left(f_{1}, f_{3}\right)$, where $\operatorname{deg}_{y}(T(x, y))=k$. Then (22) implies that $k=2$ if $s>3$, and $k=2,4$ if $s=3$. Consequently, $\operatorname{deg}_{y}\left(\frac{\partial T}{\partial y}(x, y)\right)=k-1 \in\{1,3\}$, and (2) yields

$$
\operatorname{deg}\left(\frac{\partial T}{\partial y}\left(f_{1}, f_{3}\right)\right) \geq n s .
$$

By (13),

$$
\left[f_{1}, g_{2}\right]=\left[f_{1}, f_{2}\right]+\left[f_{1}, f_{3}\right] \frac{\partial T}{\partial y}\left(f_{1}, f_{3}\right) .
$$

Assume first that $\operatorname{deg} g_{2}<n(s-2)+\operatorname{deg}\left[f_{1}, f_{3}\right]$. Then (28) yields

$$
\operatorname{deg}\left[f_{1}, f_{2}\right] \geq \operatorname{deg}\left[f_{1}, f_{3}\right]+n s .
$$

Therefore, $\operatorname{deg} f_{2} \geq n(s-2)+\operatorname{deg}\left[f_{1}, f_{3}\right]>n$ and $\bar{f}_{1} \notin\left\langle\bar{f}_{2}\right\rangle$. Consequently, either the elements $\bar{f}_{1}, \bar{f}_{2}$ are algebraically independent or $f_{1}, f_{2}$ form a $*$-reduced pair. Since $\operatorname{deg}\left[f_{1}, f_{2}\right]>n s \geq \operatorname{deg} a$, we have by Lemma 2 ii) and Corollary 1

$$
a=\alpha f_{2}^{2}+\gamma f_{2}+G\left(f_{1}\right), \quad \operatorname{deg}\left(G\left(f_{1}\right)\right)<\operatorname{deg} f_{3},
$$

where $\alpha \neq 0$ only if $\operatorname{deg} f_{3} \geq 2 \operatorname{deg} f_{2}$. Since $f_{1}, f_{3}$ is a 2-reduced pair, then $f_{1}, f_{3}+G\left(f_{1}\right)$ is also a 2 -reduced pair. By Lemma $9\left(f_{1}, g_{2}, f_{3}+G\left(f_{1}\right)\right)$ is a simple automorphism. We have

$$
\left(f_{1}, g_{2}, f_{3}+G\left(f_{1}\right)\right) \rightarrow\left(f_{1}, f_{2}, f_{3}+G\left(f_{1}\right)\right) \rightarrow\left(f_{1}, f_{2}, f_{3}+\alpha f_{2}^{2}+\gamma f_{2}+G\left(f_{1}\right)\right)=\tau .
$$

If $(\alpha, \gamma) \neq(0,0)$, then it is easily checked that $\left(f_{1}, g_{2}, f_{3}+G\left(f_{1}\right)\right)$ is a reduction of $\tau$ of types I-III, with an active element $f_{2}$. Note that the type of the reduction depends on the degree of $f_{2}$ :

1) if $2 n<\operatorname{deg} f_{2}$, then $\operatorname{deg}\left(f_{2}^{2}\right)>n(s-2)+\operatorname{deg}\left[f_{1}, f_{3}\right]+2 n>n s=\operatorname{deg} f_{3}$, $\alpha=0$, and $\tau$ admits a reduction of type I;

2) if $\frac{3 n}{2}<\operatorname{deg} f_{2} \leq 2 n$, then $s=3$ (otherwise $f_{2}$ is not reducible in $\theta$ ), $\alpha=0$ and $\tau$ admits a reduction of type II;

3 ) if $\operatorname{deg} f_{2} \leq \frac{3 n}{2}$, then $s=3$ and $\tau$ admits a reduction of type III. 
The case $\alpha=\gamma=0$ follows from Lemma 14

It remains to consider the cases when $\phi$ satisfies one of the conclusions 2), 3) of Proposition 4. If $\phi$ satisfies 3), then (28) again gives (29). Besides, in this case $s=3$ and $\operatorname{deg} f_{2}>\operatorname{deg} g_{2}=\frac{3 n}{2}$. Consequently, $a=\beta f_{2}+\gamma f_{1}$. By Lemma 14 we can put $\beta \neq 0$. Then the automorphism $\left(f_{1}, g_{2}, f_{3}+\gamma f_{1}\right)$ gives a reduction of type I or II of $\tau$ with an active element $f_{2}$.

Assume finally that $\phi$ admits a reduction of type IV with an active element $g_{2}$. Since $\operatorname{deg}\left[f_{1}, f_{3}\right] \leq 2 n$, the scalars $\alpha, \beta, \gamma$ in Definition 4 are 0 by Proposition 35 ). Consequently, the reduction of type IV of $\phi$ has the form $\left(f_{1}, h_{2}, f_{3}-\delta h_{2}^{2}\right)$, where

$$
g_{2}=h_{2}+g, g \in\left\langle f_{1}, f_{3}\right\rangle \backslash F, \quad \operatorname{deg}\left(f_{3}-\delta h_{2}^{2}\right) \leq 2 n .
$$

Since $\operatorname{deg} g_{2} \leq \operatorname{deg} h_{2}=\frac{3 n}{2}$, then $\operatorname{deg} g \leq \frac{3 n}{2}$. Note that, by Definition 4 , the automorphism $\left(f_{1}, h_{2}, f_{3}\right)$ satisfies statement 3$)$ of Proposition 4

If $\operatorname{deg} f_{2}>\frac{3 n}{2}$, then we may take $\phi=\left(f_{1}, h_{2}, f_{3}\right)$, and this case may be reduced to the previous one.

Assume that $\operatorname{deg} f_{2} \leq \frac{3 n}{2}$. Then we write

$$
f_{2}=g_{2}-b=h_{2}+(g-b), \quad g-b \in\left\langle f_{1}, f_{3}\right\rangle .
$$

If $g-b \notin F$, then $\theta$ admits the reduction of type IV $\left(f_{1}, h_{2}, f_{3}-\delta h_{2}^{2}\right)$, with an active element $f_{2}, \alpha=\beta=\gamma=0$, and the predreduction $\left(f_{1}, h_{2}, f_{3}\right)$. This case was considered in Lemma 12. If $g-b=\alpha \in F$, then $f_{2}=h_{2}+\alpha$ and the automorphism $\left(f_{1}, f_{2}, f_{3}-\delta h_{2}^{2}\right)$ is simple by Remark 1. Consequently, $f_{3}$ is a simple reducible element of $\theta$, a contradiction.

Lemma 16. If $f_{1}, f_{3}$ is a $*$-reduced pair and $\operatorname{deg} f_{3}<\operatorname{deg} f_{1}$, then $\tau$ is a simple automorphism.

Proof. Consider first the case when $\operatorname{deg} f_{1}<\operatorname{deg} f_{2}$. Since $\operatorname{deg} a \leq \operatorname{deg} f_{3}$ by (11), in this case $\bar{a} \notin\left\langle\bar{f}_{1}, \bar{f}_{2}\right\rangle$. Then the inclusion $a \in\left\langle f_{1}, f_{2}\right\rangle$ implies by F2) that the elements $\bar{f}_{1}, \bar{f}_{2}$ are algebraically dependent. By Lemma 14, we may assume that $f_{1}, f_{2}$ form a $*$-reduced pair. Since $\operatorname{deg} a<\operatorname{deg} f_{1}$, inequality (2) gives that $\operatorname{deg} f_{1}=$ $2 n, \operatorname{deg} f_{2}=3 n, \operatorname{deg}\left[f_{1}, f_{2}\right] \leq 2 n$. Besides, if $\operatorname{deg} f_{3}<n+\operatorname{deg}\left[f_{1}, f_{2}\right]=N\left(f_{1}, f_{2}\right)$, then Corollary 1 implies $a \in F$. Thus, we may assume that

$$
\operatorname{deg} f_{3} \geq n+\operatorname{deg}\left[f_{1}, f_{2}\right] .
$$

Observe that $\theta$ satisfies all the conditions of Proposition 4 hence it should satisfy one of conlusions 2), 3) of this proposition. The case when $\theta$ admits a reduction of type IV was considered in Lemma 12. Therefore, we may assume that $\operatorname{deg} f_{3}=\frac{3 n}{2}$, and

$$
\operatorname{deg}\left(f_{2}-\alpha f_{3}^{2}\right) \leq 2 n, \quad \operatorname{deg}\left[f_{1}, f_{3}\right]<3 n+\operatorname{deg}\left[f_{1}, f_{2}\right] .
$$

Observe that $\operatorname{deg} a, \operatorname{deg} f \leq \frac{3 n}{2}$. By Lemma 13, the automorphism $\left(f_{1}, f_{2}-\alpha f_{3}^{2}, f_{3}\right)$ is simple. If $a \notin F$, then $\left(f_{1}, f_{2}-\alpha f_{3}^{2}, f_{3}\right)$ gives a reduction of type IV of $\tau$.

Consider now the case when $\operatorname{deg} f_{2} \leq \operatorname{deg} f_{1}$. If $\operatorname{deg} f_{1}=\operatorname{deg} f_{2}$, then by Lemma 14 we may assume that $\bar{f}_{1}, \bar{f}_{2}$ are linearly independent. Suppose $\bar{f}_{2} \in\left\langle\bar{f}_{3}\right\rangle$. If $\bar{f}_{2}, \bar{f}_{3}$ are linearly dependent, sequence (15) proves that $\tau$ is simple. Suppose that $\operatorname{deg} f_{2}=t \cdot \operatorname{deg} f_{3}, t \geq 2$. Since $\operatorname{deg} f_{3}<\min \left\{\operatorname{deg} f_{1}, \operatorname{deg} f_{2}\right\}$ and $\operatorname{deg} a \leq \operatorname{deg} f_{3}$, under the assumption that $a \notin F$, inequality (2) yields $\operatorname{deg} f_{2}=2 n, \operatorname{deg} f_{1}=3 n$, and

$$
\operatorname{deg} f_{3} \geq n+\operatorname{deg}\left[f_{1}, f_{2}\right]>\frac{2 n}{t}=\operatorname{deg} f_{3} .
$$


Therefore, $\bar{f}_{2} \notin\left\langle\bar{f}_{1}, \bar{f}_{3}\right\rangle$. Since $f_{2}$ is a simple reducible element of $\theta$, it follows from Corollary 1 that $N\left(f_{1}, f_{3}\right) \leq \operatorname{deg} f_{2} \leq \operatorname{deg} f_{1}$ and by Lemma 2.iii) the pair $f_{3}, f_{1}$ is 2-reduced. Without loss of generality, we can assume $g_{2}$ in (13) to be unreducible in $\phi=\left(f_{1}, g_{2}, f_{3}\right)$; then $\phi$ satisfies the conditions of one of Propositions 4 5. The rest of the proof can now be fulfilled similarly to that of Lemma15.

Lemma 17. If $\bar{f}_{1} \in\left\langle\bar{f}_{3}\right\rangle$ and $\operatorname{deg} f_{1}>\operatorname{deg} f_{3}$, then $\tau$ is a simple automorphism.

Proof. Put $n=\operatorname{deg} f_{3}, \bar{f}_{1}=\alpha \bar{f}_{3}{ }^{k}, k \geq 2$. Then $\operatorname{deg} f_{1}=n k$. Consider the simple automorphism $\phi=\left(f_{1}, g_{2}, f_{3}\right)$ defined by (13). Since $\operatorname{deg} \phi<\operatorname{deg} \theta$, Lemma 9 implies that $\psi=\left(f_{1}-\alpha f_{3}^{k}, g_{2}, f_{3}\right)$ is also a simple automorphism. Therefore, by Lemma 9 again, the sequence

$$
\psi \rightarrow\left(f_{1}-\alpha f_{3}^{k}, f_{2}, f_{3}\right) \rightarrow \theta
$$

proves that $f_{1}$ is a simple reducible element of $\theta$. Interchanging the elements $f_{1}, f_{2}$, by Lemmas 14, 15, 16, we can restrict ourselves to the case when $\left.\bar{f}_{2} \in\left\langle\bar{f}_{3}\right\rangle, \operatorname{deg} f_{2}\right\rangle$ $\operatorname{deg} f_{3}$. Thus, we can take $\operatorname{deg} f_{2}=n r, r \geq 2$. By Lemma 14, we can assume that $f_{1}, f_{2}$ is a $*$-reduced pair, with $\operatorname{deg} f_{1}=m p, \operatorname{deg} f_{2}=m s,(p, s)=1, m \geq n$. Then $N\left(f_{1}, f_{2}\right)>m(p s-p-s)>n \geq \operatorname{deg} a$. Hence by Corollary $1, \bar{a} \in\left\langle\bar{f}_{1}, \bar{f}_{2}\right\rangle$, which evidently implies that $a \in F$.

This finishes the proof of Theorem [1

\section{The MAIN RESUlts}

Theorem 1 implies immediately

Theorem 2. Let $\theta=\left(f_{1}, f_{2}, f_{3}\right)$ be a tame automorphism of the ring of polynomials $A=F\left[x_{1}, x_{2}, x_{3}\right]$ over a field $F$ of characteristic 0 . If $\operatorname{deg} \theta>3$, then $\theta$ admits either an elementary reduction or a reduction of types $I-I V$.

Corollary 8. Under the conditions of Theorem Q, if $f_{3}=x_{3}$, then $\theta$ admits an elementary reduction.

Proof. In fact, it is easy to see that in this case $\theta$ does not admit reductions of types I-IV.

Now we will consider the Nagata automorphism $\sigma=(f, g, h)$ (see [12]) of the polynomial ring $F[x, y, z]$, where

$$
f=x+w z, \quad g=y+2 w x+w^{2} z, \quad h=z, \quad w=x^{2}-y z .
$$

Corollary 9. The Nagata automorphism of the polynomial ring $F[x, y, z]$ over a field $F$ of characteristic 0 is wild.

Proof. Note that $\bar{f}=w z, \bar{g}=w^{2} z, \bar{h}=z$ are mutually algebraically independent, and none of the elements $\bar{f}, \bar{g}, \bar{h}$ is contained in the subalgebra generated by the other two elements. Consequently, the automorphism $\sigma$ does not admit an elementary reduction. By Corollary 8 is wild.

In [7, some examples of wild automorphisms of the algebra $F[z][x, y]$ were constructed. The next corollary shows that all those automorphisms are also wild as automorphisms of the algebra $F[x, y, z]$. 
Corollary 10. Let $F$ be a field of characteristic 0. An automorphism $(f, g)$ of the $F[z]$-algebra $F[z][x, y]$ is tame if and only if the automorphism $(f, g, z)$ of the $F$-algebra $F[x, y, z]$ is tame.

The proof follows easily from Corollary 8

Theorem 3. The tame and the wild automorphisms of the algebra $F\left[x_{1}, x_{2}, x_{3}\right]$ of polynomials in three variables over a constructive field $F$ of characteristic 0 are algorithmically recognizable.

Proof. By induction on degree, it suffices to recognize, for every automorphism $\theta=\left(f_{1}, f_{2}, f_{3}\right)$ with $\operatorname{deg} \theta>3$, whether $\theta$ admits either an elementary reduction or a reduction of types I-IV. By Lemma 8 the elementary reducibility of $\theta$ is algorithmically recognizable.

Suppose that $\theta$ admits a reduction of types I-IV. If we assume that $f_{3}$ is an active element of the reduction and $\operatorname{deg} f_{1} \leq \operatorname{deg} f_{2}$, then the roles of the elements of $\theta$ are defined uniquely. It follows from Propositions 13 that the coefficients $\alpha, \beta, \gamma$ and the elements $g_{1}, g_{2}$ satisfying the conditions of these propositions are uniquely defined and can be found effectively. Put $\phi=\left(g_{1}, g_{2}, f_{3}\right)$.

If the element $f_{3}$ of $\phi$ is unreducible, then it remains to check whether $\phi$ admits or not a reduction of type IV with an active element $f_{3}$. By Corollary 2 the element $g_{3}$ satisfying the condition of Proposition 3 will be defined effectively by the conditions

$$
\begin{array}{r}
g_{3}=f_{3}+\delta w\left(g_{1}, g_{2}\right), \quad \delta \neq 0, \\
\operatorname{deg}\left[g_{1}, g_{3}\right]<\operatorname{deg} g_{2}+\operatorname{deg}\left[g_{1}, g_{2}\right],
\end{array}
$$

where $w(x, y)$ is a derivative polynomial of the pair $g_{1}, g_{2}$. Now it is easy to check the existence of $\mu \in F$ such that $\operatorname{deg}\left(g_{2}-\mu g_{3}^{2}\right) \leq \operatorname{deg} g_{1}$.

If $f_{3}$ is a reducible element of $\phi$, then by Lemma 8, we can effectively find an unreducible reduction $g_{3}$ of $f_{3}$. If

$$
\operatorname{deg} g_{3}<\operatorname{deg} g_{2}-\operatorname{deg} g_{1}+\operatorname{deg}\left[g_{1}, g_{2}\right],
$$

then $\psi=\left(g_{1}, g_{2}, g_{3}\right)$ is a reduction of types I, II, III of $\theta$. Otherwise it remains to check the validity of statements 2), 3) of Proposition 4 for $\psi$. It was already shown above that it can be done effectively. Then, as in the proof of Lemmas 15, 16, one can define which type of reduction $\theta$ admits.

Note that a reduction of type I consists of two elementary transformations, reductions of types II, III consist of three elementary transformations, and a reduction of type IV in general case consists of four elementary transformations. Then it follows from Theorem 2 that the degree of any tame nonlinear automorphism of $A$ can be reduced by at most four elementary transformations. In this context, the following question seems very interesting.

Problem 1. Construct examples of tame automorphisms of the algebra $A$ that admit reductions of types II-IV.

In other words, do there exist tame automorphisms of $A$ whose degrees cannot be reduced by two (or even by three) elementary transformations? The automorphism $\left(f_{1}, f_{2}, f_{3}\right)$ from Example 1 admits a reduction of type I and so its degree can be reduced by two (but not by one!) elementary transformations. 


\section{ACKNOWLEDGMENTS}

The second author is grateful to the Institute of Mathematics and Statistics of the University of Sao Paulo for its warm hospitality during his visit from October 9, 2000 until August 31, 2001 when the main part of this work was done.

We are grateful to L. Makar-Limanov, A. A. Mikhalev, V. Shpilrain, and J.-T. $\mathrm{Yu}$ for thoroughly going over the details of the proofs during an informal seminar at the University of Hong Kong in June 2002. We also thank A. van den Essen, D. Wright, and E. Zelmanov for numerous helpful comments.

\section{REFERENCES}

[1] A. T. Abdykhalykov, A. A. Mikhalev, U. U. Umirbaev, Automorphisms of two generated free Leibniz algebras, Commun. Algebra. 29 (2001), 2953-2960. MR 2002e:17004

[2] H. Bass, Automorphisms of Polynomial Rings, Lecture Notes in Math., 1006, Springer-Verlag, Berlin, pp. 762-771. MR 85b:13009

[3] H. Bass, A non-triangular action of $G_{a}$ on $A^{3}$, J. of Pure and Appl. Algebra 33 (1984), no. $1,1-5$.

[4] G. M. Bergman, Wild automorphisms of free P.I. algebras and some new identities, preprint.

[5] P. M. Cohn, Free rings and their relations, 2nd edition, Academic Press, London, 1985. MR 87e:16006

[6] A. G. Czerniakiewicz, Automorphisms of a free associative algebra of rank 2, I, II, Trans. Amer. Math. Soc. 160 (1971), 393-401; 171 (1972), 309-315. MR 43:6269 MR 46:9124

[7] V. Drensky, J.-T. Yu, Tame and wild coordinates of $K[z][x, y]$, Trans. Amer. Math. Soc. 353 (2001), no. 2, 519-537. MR 2001f:13028

[8] A. van den Essen, Polynomial automorphisms and the Jacobian conjecture, Progress in Mathematics, 190, Birkhäuser-Verlag, Basel, 2000. MR 2001j:14082

[9] H. W. E. Jung, Über ganze birationale Transformationen der Ebene, J. reine angew. Math. 184(1942), 161-174. MR 5:74f

[10] W. van der Kulk, On polynomial rings in two variables, Nieuw Archief voor Wiskunde. (3) 1 (1953), 33-41. MR 14:941f

[11] L. Makar-Limanov, The automorphisms of the free algebra of two generators, Funksional. Anal. i Prilozhen. 4 (1970), no. 3, 107-108. MR 42:6044

[12] M. Nagata, On the automorphism group of $k[x, y]$, Lectures in Math., Kyoto Univ., Kinokuniya, Tokyo, 1972. MR 49:2731

[13] G. A. Noskov, The cancellation problem for a ring of polynomials, Sibirsk. Mat. Zh. 19 (1978), no. 6, 1413-1414. MR 81g:13005

[14] D. Shannon, M. Sweedler, Using Gröbner bases to determine algebra membership, split surjective algebra homomorphisms determine birational equivalence, J. Symbolic Comput. 6 (1988), 267-273. MR 90e:13002

[15] I. P. Shestakov, Quantization of Poisson superalgebras and speciality of Jordan Poisson superalgebras, Algebra i logika, 32 (1993), no. 5, 571-584; English translation: in Algebra and Logic, 32 (1993), no. 5, 309-317. MR 95c:17034

[16] I. P. Shestakov, U. U. Umirbaev, Poisson brackets and two-generated subalgebras of rings of polynomials, J. Amer. Math. Soc. 17 (2004), 181-196.

[17] M. K. Smith, Stably tame automorphisms, J. Pure and Appl. Algebra 58 (1989), 209-212. MR 90f:13005

[18] U. U. Umirbaev, Universal derivations and subalgebras of free algebras, In Proc. 3rd Internat. Conf. in Algebra (Krasnoyarsk, 1993). Walter de Gruyter, Berlin, 1996, 255-271. MR 97c:16030

[19] D. Wright, Algebras which resemble symmetric algebras, Ph.D. Thesis, Columbia Univ., New York, 1975. 
Instituto de Matemática e Estatística, Universidade de São Paulo, Caixa Postal 66281, São Paulo - SP, 05311-970, Brazil; Sobolev Institute of Mathematics, NovosiBIRSK, 630090, RUSSIA

E-mail address: shestak@ime.usp.br

Department of Mathematics, Eurasian National University, Astana, 473021, KazaKHSTAN

E-mail address: umirbaev@yahoo.com 